\title{
Using TopGear in Overdrive: A more efficient ZKPoK for SPDZ
}

\author{
Carsten Baum ${ }^{1[0000-0001-7905-0198]}$ Daniele Cozzo $\left.20000-0001-5289-3769\right]$ and Nigel P. \\ Smart $^{2,3}[0000-0003-3567-3304]$ \\ 1 Aarhus University, Denmark. \\ 2 imec-COSIC, KU Leuven, Leuven, Belgium. \\ ${ }^{3}$ University of Bristol, Bristol, UK. \\ cbaum@cs.au.dk, daniele.cozzo@kuleuven.be, nigel.smart@kuleuven.be
}

\begin{abstract}
The HighGear protocol (Eurocrypt 2018) is the fastest currently known approach to preprocessing for the SPDZ Multi-Party Computation scheme. Its backbone is formed by an Ideal Lattice-based Somewhat Homomorphic Encryption Scheme and accompanying Zero-Knowledge proofs. Unfortunately, due to certain characteristics of HighGear such current implementations limit the security parameters in a number of places. This is mainly due to memory and bandwidth consumption constraints.

In this work we present a new approach to the ZKPoKs for the SPDZ Multi-Party Computation scheme. We rigorously formalize the original approach of HighGear and show how to improve upon it using a different proof strategy. This allows us to increase the security of the underlying protocols, whilst simultaneously also increasing the performance in terms of memory and bandwidth consumption as well as overall throughput of the SPDZ offline phase.
\end{abstract}

\section{Introduction}

Multi-party computation (MPC) has turned in the last fifteen years from a mainly theoretical endeavor to one which is now practical, with a number of companies fielding products based on it. MPC comes in a number of flavours, depending on the underlying primitives (garbled circuits, secret sharing), number of parties (two or many parties) and security model (passive, covert, active). In this work we will focus on $n$-party secret sharing-based MPC for arithmetic circuits, secure against an active adversary corrupting a dishonest majority of parties. In this setting the most efficient protocol known is the SPDZ protocol [15] from 2012, along with its many improvements such as [14|24|25].

The SPDZ protocol family uses a form of authenticated secret sharing over a finite field $\mathbb{F}_{p}$ to perform the secure computation. The protocol is divided into two phases, an offline phase (which produces, among other things, multiplication triples) and an online phase (which usese these preprocessed multiplication triples to perform actual multiplications). In this work we will be focusing on the offline phase, specifically on how to increase its security level while keeping or increasing the performance. The SPDZ protocol, among others, has been implemented in the SCALE-MAMBA system [2], which we refer to as a reference implementation allowing to measure and compare our contribution in practice.

To understand the difference of our approach in terms of efficiency and security we first consider the genesis of the problem we solve. The SPDZ protocol is itself based on an earlier work called BDOZ [7]. The BDOZ protocol used a form of pairwise MACs to authenticate a secret sharing amongst $n$-parties. At the heart of the offline phase for BDOZ is a pairwise multiplication protocol, using linearly homomorphic encryption. To ensure that active adversaries do not cheat in this phase pairwise zero-knowledge proofs are utilized to ensure active adversaries cannot deviate from the protocol without detection. In total $O\left(n^{2}\right)$ ZKPoKs need to be carried out per multiplication triple of BDOZ.

The main contribution of the SPDZ paper [15] over BDOZ was to replace the pairwise MACs with a global MAC. This was made possible by upgrading the linearly homomorphic encryption used in BDOZ to a limited form of Somewhat Homomorphic Encryption (SHE) based on the BGV encryption scheme [10]. This new scheme permits to reduce the number of ZK proofs per multiplication triple by a factor of $n$. At the same time, due to the SmartVercauteren SIMD packing underlying BGV plaintext spaces the overhead due to the ZKPoKs per multiplication triple was drastically reduced. This is because a single ZKPoK could be used to simultaneously prove statements for many thousands of multiplication triples. 
However, BGV is a lattice based SHE scheme and ZKPoKs for these are relatively costly - due to the necessity of proving bounds on the size of plaintexts and randomness. For a single ciphertext, the basic $\Sigma$-protocol has challenge space $\{0,1\}$ and must thus be repeated many times to achieve reasonable security levels. Furthermore, to provide zero-knowledge one needs to "blow-up" the proven bounds, so the proven statement is strictly weaker than the honest parameter choice $\rfloor^{4}$ This introduces what is called the soundness slack between the honest language $\mathbb{L}$ and the proven language $\mathbb{L}^{\prime}$.

To get around the problem of having to perform multiple proofs for the same ciphertext, SPDZ uses a standard amortization technique [12] to prove $U$ statements at once, where $U$ is the number of ciphertexts used in the protocol. This boosts the soundness security from $1 / 2$ to $2^{-U}$, at the expense of introducing even more soundness slack, namely an additional factor of $2^{U / 2}$. At the same time, [12] crucially needs to send $V=2 \cdot U-1$ auxiliary ciphertexts. In the implementation of this ZKPoK in the original, and subsequent works, the authors set $U$ to be the same security level as the statistical zero-knowledge parameter ZK_sec.

Despite this use of amortization techniques, the ZKPoKs were for a long time considered too slow. This resulted in two new techniques based on cut-and-choose (being introduced in [14]). The first of these produced only covert security, but was highly efficient, and thus for a number of years all implementations of the SPDZ offline phase only provided covert security. The second approach of [14] provided actively secure ZKPoK which seemed asymptotically more efficient than those provided in [15], but which due to large memory requirements were impossible to implement.

This inability to provide efficient actively secure offline phased based on SHE led to a temporary switch to an Oblivious Transfer-based offline phase, called MASCOT [24]. However, in 2018 Keller et al. [25] introduced the Overdrive suite of offline protocols. There, they revisited the original SPDZ offline phase and showed two interesting ways to optimize it. Firstly, in so-called LowGear, for a small number of parties the original pairwise ZKPoKs of the BDOZ methodology could be more efficient than that of SPDZ. They observe that an $O\left(n^{2}\right)$ algorithm can beat an $O(n)$ algorithm for small values of $n$ and in addition improve the parameters of the SHE scheme. This was partially also enabled by using the same SIMD packing in BDOZ as was being used for SPDZ.

The second variant of Overdrive, called HighGear, works for larger values of $n$. Here, the original SPDZ ZKPoK was revisited and tweaked. While previously the ZKPoK was used so each party proved a statement to each other party, in HighGear the parties proved a single joint statement together for their secret inputs and an accumulated ciphertext. This did not provide an improvement in communication efficiency, but it did make the computational costs a factor of $n$ smaller.

In the SCALE-MAMBA system (as of v1.2 in Nov 2018) only the HighGear variant of Overdrive is implemented for the case of dishonest majority MPC, even when $n=2$. However, like all prior work the system adopts $U=Z$ Z _sec, and thus achieves the same soundness security Snd_sec as zero-knowledge indistinguishability. This is neither as efficient, nor as secure, as one would want for two combined reasons.

1. The zero-knowledge security ZK_sec is related to a statistical distance, whereas the soundness security Snd_sec is related to the probability that an adversary can cheat in an interactive protocol. A low value for Snd_sec is rather more acceptable than a low value for ZK_sec.

2. The practical complexity of the protocol, in particular the memory and computational consumption, is dominated by Snd_sec. It turns out that ZK_sec has very virtually no effect on the overall execution time of the offline phase, for large values of $p$.

It is for this reason that [25] gives performance metrics for 40, 64 and 128-bit active security, and why v1.2 of SCALEMAMBA utilizes only 40-bits of security for Snd_sec and ZK_sec, since the execution time is highly dependent on Snd_sec.

Our Contribution. We first formalize the type of statement which the Overdrive ZKPoK tries to prove. The original treatment in [25] is relatively intuitive. We formalize the statement by presenting a generalization of standard Zero Knowledge-protocols to what we call an $n$-party Zero Knowledge-protocol. The formalization is tailored at the use of such proofs in preprocessing.

\footnotetext{
${ }^{4}$ There do exist ZKPoKs for lattice-based primitives which prove exact bounds. Unfortunately, their computation and communication overhead makes them no match in practice for protocols having soundness slack.
} 
We then present a modified ZKPoK for the HighGear variant of Overdrive, which we denote TopGear. It treats the soundness security Snd_sec and the zero-knowledge security ZK_sec separately. This ZKPoK in its unamortized variant (i.e. only proving one statement at a time) uses a non-binary challenge space in a similar way as was done in [8]. Hence we obtain a challenge space of $2 \cdot N+1$ in the "base" ZKPoK (where $N$ is the ring dimension of the cryptosystem used). We then amortize this base ZKPoK by proving $U$ statements in parallel using a technique from [3]. This enables us to achieve an arbitrary knowledge soundness of Snd_sec by selecting the number of auxiliary ciphertexts $V$ such that $V \geq($ Snd_sec +2$) / \log _{2}(2 \cdot N+1)$.

Since $N$ is often $32768=2^{15}$, we are able to achieve a high soundness security, with a low value of $V$, e.g. we can obtain 128 bits of soundness security by setting $V$ to be 8 . This translates into a smaller amount of amortization than [25], and thus a smaller memory footprint and bandwidth for the same level of ZK_sec. Alternatively, we can select higher values of ZK_sec, if so desired, as this has little impact on the overall performance.

Concerning the slack we follow an approach similar to [25] but with a twist. In signature schemes based on lattices, such as BLISS, this issue is usually dealt with using rejection sampling, e.g. [18[19]26]. As the slack in TopGear will be removed due to the processing that happens after the ZKPoK is executed (during a modulus switch operation in the SHE scheme), we start with a simpler yet more efficient technique to achieve zero-knowledge called "noise drowning". We generally obtain a smaller slack and thus better SHE parameters due to the change of the ZKPoK from [12] to [3]. Due to our use of the larger challenge space of [8] we also cannot extract an "exact" preimage in the soundness proof, but only that of a related ciphertext. We will show that this can be corrected easily in the case of SPDZ preprocessing.

Other Related Work. Multiple techniques have been introduced to cope with the problem of amortized ZKPoK for lattice-based primitives. Multiple subsequent works [4[13|16] have introduced more and more efficient proofs of knowledge which have small (down to linear in Snd_sec) slack. Unfortunately, all of these require $U$ to be in the multiple of 1000s to be efficient, which is far from practically feasible. Later, Baum and Lyubashevsky [5] showed how to build small-slack proofs for realistic sizes of $U$, though their idea was limited to structured lattices. The problem was only recently resolved in [3] which we therefore use as a building block in our work.

Another recent methodology to perform such ZKPoKs as needed in this paper is given in [17] based on bullet proofs. These give very short proofs but are not competitive with the approach in this paper. Firstly, we use amortization over the number of parties to produce a joint proof whereas the direct application of [17] would require pairwise proofs which would not scale well with the number of parties. The paper [17] also concentrates on the case of "small moduli $q$ " of the SHE ciphertext space. In our case, we easily need this $q$ to have a size of $>500$ bits. Apart from the problem of constructing a secure DLP group of a given order at this size (leading to probably needing to use finite fields, or a group size larger than $q$ to deal with integer overflow), this also leads to very large computational expenses. To sign and verify a proof from [17] requires at least $12 \cdot N \cdot \log _{2} q$ exponentiations (where $N$ again is the dimension of the rings used in the ideal lattice-based cryptosystem). In our case this would equate to around $2^{27}$ exponentiations for each proof.

\section{Preliminaries}

In this section we provide a recap of the the BGV encryption scheme [10] as well as those building blocks of SPDZ that are used in combination with it. Most of the details about BGV can be found in [10|21|22]20], although we will employ a variant which supports circuits of multiplicative depth one only.

Notation. We generally let $P_{i}$ denote a party, of which there are $n$ in total. Those parties are modeled as probabilistic polynomial time (PPT) Turing machines. We let $[n]$ denote the interval $[1, \ldots, n]$. If $M$ is a matrix then we write $M^{(r, c)}$ for the entry in the $r$-th row and $c$-th column. Vectors are (usually) written in bold, and their elements in nonbold with a subscript, thus $\boldsymbol{x}=\left(x_{i}\right)_{i \in[n]}$. We will write $\boldsymbol{x}[i]$ to denote the $i$-th element in the vector $\boldsymbol{x}$. All modular reduction operations $x(\bmod q)$ will be to the centered interval $(-q / 2, q / 2]$.

We let $a \leftarrow X$ denote randomly assigning a value $a$ from a set $X$, where we assume a uniform distribution on $X$. If $A$ is an algorithm, we let $a \leftarrow A$ denote assignment of the output, where the probability distribution is over the random tape of $A$; we also let $a \leftarrow b$ be a shorthand for $a \leftarrow\{b\}$, i.e. to denote normal variable assignment. If $\mathcal{D}$ is a probability distribution over a set $X$ then we let $a \leftarrow \mathcal{D}$ denote sampling from $X$ with respect to the distribution $\mathcal{D}$. 
We will make use of the following standard lemma in a number of places

Lemma 1. Let $\mathcal{D}$ be any distribution whose values are bounded by $B$. Then the distributions $\mathcal{D}+\mathcal{U}\left(0, \cdots, B^{\prime}\right)($ by which we mean the distribution obtained from sampling from the two distributions and adding the result) is statistically close to the uniform distribution $\mathcal{U}\left(0, \cdots, B^{\prime}\right)$, with statistical distance bounded by $\frac{B}{B^{\prime}}$.

SPDZ Secret Sharing. This SPDZ protocol [15] processes data using an authenticated secret sharing scheme defined over a finite field $\mathbb{F}_{p}$, where $p$ is prime. The secret sharing scheme is defined as follows: Each party $P_{i}$ holds a share $\alpha_{i} \in \mathbb{F}_{p}$ of a global MAC key $\alpha=\sum_{i \in[n]} \alpha_{i}$. A data element $x \in \mathbb{F}_{p}$ is held in secret shared form as a tuple $\left\{x_{i}, \gamma_{i}\right\}_{i \in[n]}$, such that $x=\sum_{i} x_{i}$ and $\sum \gamma_{i}=\alpha \cdot x$. We denote a value $x$ held in such a secret shared form as $\langle x\rangle$. The main goal of the SPDZ offline phase is to produce random triples $(\langle a\rangle,\langle b\rangle,\langle c\rangle)$ such that $c=a \cdot b$. If we wish to denote the specific value on which $\gamma_{i}$ is a MAC share then we write $\gamma_{i}[x]$.

The Rings. The BGV encryption scheme, as we will use it, is built around the arithmetic of the cyclotomic ring $\mathcal{R}=\mathbb{Z}[X] /\left(\Phi_{m}(X)\right)$, where $\Phi_{m}(X)$ is the $m$-th cyclotomic polynomial. For an integer $q>0$, we denote by $\mathcal{R}_{q}$ the ring obtained as reduction of $\mathcal{R}$ modulo $q$. We take $m$ to be a power of two, $m=2^{n+1}$ and hence $\Phi_{m}(X)=X^{N}+1$ where $N=2^{n}$. Elements of $\mathcal{R}$ (resp. $\mathcal{R}_{q}$ ) can either be thought of as polynomials (of degree less than $N$ ) or as vectors of elements (of length $N$ ).

The canonical embedding of $\mathcal{R}$ is the mapping of $\mathcal{R}$ into $\mathbf{C}^{\phi(m)}$ given by $\sigma(x)=\left(x\left(\zeta_{m}^{i}\right)\right)_{i \in[n]}$, where we think of $x$ as a polynomial. We are interested in two norms of elements $x$ in $\mathcal{R}$ (resp. $\mathcal{R}_{q}$ ). For the $\infty$-norm in the standard polynomial embedding we write $\|x\|_{\infty}$, whereas the $\infty$-norm in the canonical embedding we will write as $\|x\|_{\infty}^{\text {can }}=$ $\|\sigma(x)\|_{\infty}$. By standard inequalities we have $\|x \cdot y\|_{\infty}^{\mathrm{can}} \leq\|x\|_{\infty}^{\mathrm{can}} \cdot\|y\|_{\infty}^{\mathrm{can}},\|x\|_{\infty}^{\mathrm{can}} \leq\|x\|_{1},\|x\|_{\infty}^{\mathrm{can}} \leq \phi(m) \cdot\|x\|_{\infty}^{\infty}$ and $\|x\|_{\infty} \leq\|x\|_{\infty}^{\text {can }}$; with the last two inequalities holding due to our specific choice of cyclotomic ring. Such norms can also be employed on elements of $\mathcal{R}_{q}$ by using the standard (centered) embedding of $\mathcal{R}_{q}$ into $\mathcal{R}$.

We will use the following two facts in a number of places.

Lemma 2. Let $m$ be a power of two. Then, for all $0 \leq i, j<2 \cdot N$,

$$
\left\|2 \cdot\left(X^{i}-X^{j}\right)^{-1} \quad\left(\bmod \phi_{m}(X)\right)\right\|_{\infty} \leq 1
$$

Proof. Given in [8].

Lemma 3. In the ring $\mathcal{R}$ defined by $\Phi_{m}(X)$ with $m$ a power of two we have that for all $a \in \mathcal{R}$ that $\left\|a \cdot X^{i}\right\|_{\infty}=$ $\|a\|_{\infty}$.

Proof. This follows as $X^{i}$ acts as a shift operation, with the wrap-around modulo $\phi(m)$ simply negating the respective coordinate.

Distributions as used in BGV. Following [22] Full version, Appendix A.5] and [2] we use different distributions to define the BGV scheme, all of which produce vectors of length $N$ which we consider as elements in $\mathcal{R}$.

- HWT $(h, N)$ : This generates a vector of length $N$ with elements chosen at random from $\{-1,0,1\}$ subject to the condition that the number of non-zero elements is equal to $h$.

- ZO $(0.5, N)$ : This generates a vector of length $N$ with elements chosen from $\{-1,0,1\}$ such that the probability of each coefficient is $p_{-1}=1 / 4, p_{0}=1 / 2$ and $p_{1}=1 / 4$. Thus if $x \leftarrow \mathrm{ZO}(0.5, N)$ then $\|x\|_{\infty} \leq 1$.

- $\operatorname{dN}\left(\sigma^{2}, N\right)$ : This generates a vector of length $N$ with elements chosen according to an approximation to the discrete Gaussian distribution with variance $\sigma^{2}$.

- $\operatorname{RC}\left(0.5, \sigma^{2}, N\right)$ : This generates a triple of elements $\left(r_{1}, r_{2}, r_{3}\right)$ where $r_{3}$ is sampled from $\mathrm{ZO}_{s}(0.5, N)$ and $r_{1}$ and $r_{2}$ are sampled from $\mathrm{dN}_{s}\left(\sigma^{2}, N\right)$.

- $\mathrm{U}(q, N)$ : This generates a vector of length $N$ with elements generated uniformly modulo $q$ in a centred range. Thus $x \leftarrow \mathrm{U}(q, N)$ implies $\|x\|_{\infty} \leq q / 2$.

Following prior work on SPDZ we select $\sigma=3.17$ and hence we can approximate the sampling from the discrete Gaussian distribution using a binomial distribution, as is done in NewHope [1]. In such a situation an element $x \leftarrow$ $\mathrm{dN}\left(\sigma^{2}, N\right)$ is guaranteed to satisfy $\|x\|_{\infty} \leq 20$. 
The Two-Level BGV Scheme. We consider a two-leveled homomorphic scheme, given by the algorithms $\{$ KeyGen, Enc, SwitchMod, Dec $\}$. The plaintext space is the $\operatorname{ring} \mathcal{R}_{p}$, for some prime modulus $p$, which is the same modulus used to define the SPDZ secret sharing scheme. The algorithms are parametrized by a computational security parameter $\kappa$ and are defined as follows. First we fix two moduli $q_{0}$ and $q_{1}$ such that $q_{1}=p_{0} \cdot p_{1}$ and $q_{0}=p_{0}$, where $p_{0}, p_{1}$ are prime numbers. Encryption generates level one ciphertexts, i.e. with respect to the largest modulo $q_{1}$, and level one ciphertexts can be moved to level zero ciphertexts via the modulus switching operation. We require $p_{1} \equiv 1(\bmod p)$ and $p_{0}-1 \equiv p_{1}-1 \equiv 0(\bmod p)$. The first condition is to enable modulus switching to be performed efficiently, whereas the second is to enable fast arithmetic using Number Theoretic Fourier Transforms.

The algorithms of the BGV scheme are then as follows:

- KeyGen $\left(1^{\kappa}\right)$ : The secret key $\mathfrak{s k}$ is randomly selected from a distribution with Hamming weight $h$, i.e. HWT $(h, N)$, much as in other systems, e.g. HELib [23] and SCALE [2]. The public key, pke, is of the form $(a, b)$, such that $a \leftarrow$ $\mathrm{U}\left(q_{1}, N\right)$ and $b=a \cdot \mathfrak{s k}+p \cdot \epsilon\left(\bmod q_{1}\right)$, where $\epsilon \leftarrow \mathrm{dN}\left(\sigma^{2}, N\right)$. This algorithm also outputs the relinearization data $\left(a_{\mathfrak{s k}, \mathfrak{s k}}, b_{\mathfrak{s k}, \mathfrak{s k}}\right)[11]$, where $a_{\mathfrak{s k}, \mathfrak{s k}^{2}} \leftarrow \mathrm{U}\left(q_{1}, N\right)$ and $b_{\mathfrak{s k}, \mathfrak{s k}^{2}}=a_{\mathfrak{s k}, \mathfrak{s k}^{2}} \cdot \mathfrak{s k}^{\mathfrak{k}}+p \cdot r_{\mathfrak{s k}, \mathfrak{s k}}-p_{1} \cdot \mathfrak{s k}^{2}\left(\bmod q_{1}\right)$, with $r_{\mathfrak{s k}, \mathfrak{F}^{2}} \leftarrow \mathrm{dN}\left(\sigma^{2}, N\right)$.

- Enc $(m, \boldsymbol{r} ; \mathfrak{p k})$ : Given a plaintext $m \in \mathcal{R}_{p}$, and randomness $\boldsymbol{r}=\left(r_{1}, r_{2}, r_{3}\right)$ chosen from $\operatorname{RC}\left(0.5, \sigma^{2}, n\right)$, i.e. we sample $r_{1}, r_{2} \leftarrow \mathrm{dN}\left(\sigma^{2}, N\right)$ and $r_{3} \leftarrow \mathrm{ZO}(0.5, N)$, this algorithm sets $c_{0}=b \cdot r_{3}+p \cdot r_{1}+m\left(\bmod q_{1}\right)$ and $c_{1}=a \cdot r_{3}+p \cdot r_{2}\left(\bmod q_{1}\right)$. Hence the initial ciphertext is $\mathfrak{c t}=\left(1, c_{0}, c_{1}\right)$, where the first index denotes the level (initially set to be equal to one). If the level $\ell$ is obvious we drop it in future discussions and refer to the ciphertext as an element in $\mathcal{R}_{q_{\ell}}^{2}$.

- SwitchMod $\left(\left(1, c_{0}, c_{1}\right)\right)$ : We define a modulus switching operation which allows us to move from a level one to a level zero ciphertext, without altering the plaintext polynomial, that is

$$
\left(0, c_{0}^{\prime}, c_{1}^{\prime}\right) \leftarrow \operatorname{SwitchMod}\left(\left(1, c_{0}, c_{1}\right)\right), \quad c_{0}^{\prime}, c_{1}^{\prime} \in \mathcal{R}_{q_{0}} .
$$

The effect of this operation is also to scale the noise term (see below) by a factor of $q_{0} / q_{1}=1 / p_{1}$.

- $\operatorname{Dec}\left(\left(c_{0}, c_{1}\right) ; \mathfrak{s k}\right)$ : Decryption is obtained by switching the ciphertext to level zero (if it is not already at level zero) and then decrypting $\left(0, c_{0}, c_{1}\right)$ via the equation $\left(c_{0}-\mathfrak{s k} \cdot c_{1}\left(\bmod q_{0}\right)\right)(\bmod p)$, which results in an element of $\mathcal{R}_{p}$.

Homomorphic Operations. Ciphertexts at the same level $\ell$ can be added,

$$
\left(\ell, c_{0}, c_{1}\right) \boxplus\left(\ell, c_{0}^{\prime}, c_{1}^{\prime}\right)=\left(\ell,\left(c_{0}+c_{0}^{\prime} \quad\left(\bmod q_{\ell}\right)\right),\left(c_{1}+c_{1}^{\prime} \quad\left(\bmod q_{\ell}\right)\right),\right.
$$

with the result being a ciphertext, which encodes a plaintext that is the sum of the two initial plaintexts. Ciphertexts at level one can be multiplied together to obtain a ciphertext at level zero, where the output ciphertext encodes a plaintext which is the product of the plaintexts encoded by the input plaintexts. We do not present the method here, although it is pretty standard consisting of a modulus-switch, tensor-operation, then relinearization (which we carry out in this order). We write the operation as

$$
\left(1, c_{0}, c_{1}\right) \odot\left(1, c_{0}^{\prime}, c_{1}^{\prime}\right)=\left(0, c_{0}^{\prime \prime}, c_{1}^{\prime \prime}\right), \quad \text { with } \quad c_{0}^{\prime \prime}, c_{1}^{\prime \prime} \in \mathcal{R}_{q_{0}},
$$

or more simply as $\left(c_{0}, c_{1}\right) \odot\left(c_{0}^{\prime}, c_{1}^{\prime}\right)=\left(c_{0}^{\prime \prime}, c_{1}^{\prime \prime}\right)$ as the levels are implied.

Ciphertext Noise. The noise term associated with a ciphertext is the value $\left\|c_{0}-\mathfrak{s k} \cdot c_{1}\right\|_{\infty}^{\text {can }}$. To derive parameters for the scheme we need to maintain a handle on this value. The term is additive under addition and is roughly divided by $p_{1}$ under a modulus switch. For the tensoring and relinearization in multiplication the terms roughly multiply. A ciphertext at level zero will decrypt correctly if we have $\left\|c_{0}-\mathfrak{s k} \cdot c_{1}\right\|_{\infty} \leq q_{0} / 2$, which we can enforce by requiring $\left\|c_{0}-\mathfrak{s k} \cdot c_{1}\right\|_{\infty}^{\mathrm{can}} \leq q_{0} / 2$.

We would like a ciphertext (adversarially chosen or not) to decrypt correctly with probability $1-2^{-\epsilon}$. In [22] $\epsilon$ is chosen to be around 55, but the effect of $\epsilon$ is only in producing the following constants: we define $e_{i}$ such that $\operatorname{erfc}\left(e_{i}\right)^{i} \approx 2^{-\epsilon}$ and then we set $\mathfrak{c}_{i}=e_{i}^{i}$. This implies that $\mathfrak{c}_{1} \cdot \sqrt{V}$, is a high probability bound on the canonical norm 
of a ring element whose coefficients are selected from a distribution with variance $V$, while $\mathfrak{c}_{2} \cdot \sqrt{V_{1} \cdot V_{2}}$ is a similar bound on a product of elements whose coefficient are chosen from distributions of variance $V_{1}$ and $V_{2}$ respectively.

With probability much greater than $1-2^{-\epsilon}$ the "noise" of an honestly generated ciphertext (given honestly generated keys) will be bounded by

$$
\begin{aligned}
\left\|c_{0}-\mathfrak{s k} \cdot c_{1}\right\|_{\infty}^{\text {can }}= & \|\left((a \cdot \mathfrak{s k}+p \cdot \epsilon) \cdot r_{3}+p \cdot r_{1}+m-\left(a \cdot r_{3}+p \cdot r_{2}\right) \cdot \mathfrak{s k} \|_{\infty}^{\text {can }}\right. \\
= & \left\|m+p \cdot\left(\epsilon \cdot r_{3}+r_{1}-r_{2} \cdot \mathfrak{s k}\right)\right\|_{\infty}^{\text {can }} \\
\leq & \|m\|_{\infty}^{\text {can }}+p \cdot\left(\left\|\epsilon \cdot r_{3}\right\|_{\infty}^{\text {can }}+\left\|r_{1}\right\|_{\infty}^{\text {can }}+\left\|r_{2} \cdot \mathfrak{s k}\right\|_{\infty}^{\text {can }}\right) \\
\leq & \phi(m) \cdot p / 2 \\
& +p \cdot \sigma \cdot\left(\mathfrak{c}_{2} \cdot \phi(m) / \sqrt{2}+\mathfrak{c}_{1} \cdot \sqrt{\phi(m)}+\mathfrak{c}_{2} \cdot \sqrt{h \cdot \phi(m)}\right) \\
= & B_{\text {clean }} .
\end{aligned}
$$

Recall this is the average case bound on the noise of honestly generated ciphertexts. In the preprocessing ciphertexts can be adversarially generated, and determining (and ensuring) a worst case bound on the resulting ciphertexts is the main focus of the HighGear and TopGear protocols.

Distributed Decryption. The BGV encryption scheme supports a form of distributed decryption, which is utilized in the SPDZ offline phase. A secret key $\mathfrak{s k}^{\mathrm{k}} \in \mathcal{R}_{q}$ can be additively shared amongst $n$ parties by giving each party a value $\mathfrak{s k}_{i} \in \mathcal{R}_{q}$ such that $\mathfrak{s k}^{\prime}=\mathfrak{s k}_{1}+\ldots+\mathfrak{s k}_{n}$. We assume, as is done in most other works on SPDZ, that the key generation phase, including the distribution of the shares of the secret key to the parties, is done in a trusted setup.

To perform a distributed decryption of a ciphertext $\mathfrak{c t}=\left(c_{0}, c_{1}\right)$ at level zero, each party computes $d_{i} \leftarrow c_{0}-c_{1}$. $\mathfrak{s k}_{i}+p \cdot R_{i}\left(\bmod q_{0}\right)$ where $R_{i}$ is a uniformly random value selected from $\left[0, \ldots, 2^{\text {DD_sec }} \cdot B / p\right]$ where $B$ is an upper bound on the norm $\left\|c_{0}-c_{1} \cdot \mathfrak{s k}\right\|_{\infty}$. The values $d_{i}$ are then exchanged between the players and the plaintext is obtained from $m \leftarrow\left(d_{1}+\ldots+d_{n}\left(\bmod q_{0}\right)\right)(\bmod p)$. The statistical distance between the distribution of the coefficients of $d_{i}$ and uniformly random elements of size $2^{\text {DD_sec }} \cdot B$ is bounded by $2^{- \text {DD_sec }}$ by Lemma 1 . To ensure valid decryption we need the value of $q_{0}$ to satisfy $q_{0}>2 \cdot\left(1+n \cdot 2^{\text {DD_sec }}\right) \cdot B$ instead of $q_{0}>2 \cdot B$ for a scheme without distributed decryption, which implies parameter growth in the BGV scheme when using this distributed decryption procedure.

\section{3 n-Prover Zero Knowledge-Protocols}

The Overdrive ZKPoK is an $n+1$ party protocol between $n$ provers and one verifier 5 Unlike traditional proofs of knowledge, there is a difference between the language used for completeness and the language that the soundness guarantees; much like the protocols considered in [9. Definition 2.2]. As the Overdrive paper does not formalize such proofs, our first contribution is to do precisely this. We give a generalized treatment of such proofs beyond regular $\Sigma$-protocols.

Let Samp be a PPT algorithm which, on input $n, i \in \mathbb{N}, 0<i \leq n$ outputs a pair of values $x_{i}, w_{i}$ where we consider $x_{i}$ as the public and $w_{i}$ as the private value. We require that if for all $i \in[n]$ we sample $\left(x_{i}, w_{i}\right) \leftarrow \operatorname{Samp}_{n}(i)$, then a given predicate $\mathbf{P}$ always holds, i.e. we have that $\mathbf{P}\left(x_{1}, \ldots, x_{n}, w_{1}, \ldots, w_{n}\right)=1$. The predicate $\mathbf{P}$ defines a language $\mathbb{L}$ via the binary relation on the pairs $\left(\boldsymbol{x}=\left(x_{1}, \ldots, x_{n}\right), \boldsymbol{w}=\left(w_{1}, \ldots, w_{n}\right)\right)$.

Consider a set of $n$ provers $P_{1}, \ldots, P_{n}$, each with private input $w_{i}$ and public input $x_{i}$. The provers wish to convince a verifier $V$ (that could be one or all of the provers) that, for the public values $x_{1}, \ldots, x_{n}$ they know $w_{1}, \ldots, w_{n}$ such that $\mathbf{P}$ holds. The guarantee provided by our proof is, however, only that $\mathbf{P}^{\prime}\left(x_{1}, \ldots, x_{n}, w_{1}, \ldots, w_{n}\right)=1$ for some second language $\mathbb{L}^{\prime}$, defined by a predicate $\mathbf{P}^{\prime}$, with $\mathbb{L} \subseteq \mathbb{L}^{\prime}$. This is still sufficient for the preprocessing of SPDZ.

Definition 1. An n-party ZKPoK-protocol with challenge set $\mathcal{C}$ for the languages $\mathbb{L}$, $\mathbb{L}^{\prime}$ and sampler Samp $\mathrm{p}_{n}$ is defined as a tuple of algorithms (Comm, Resp, Verify). While Comm is a PPT algorithm, we assume that Resp, Verify are deterministic. The verifier $V$ will have input $x_{1}, \ldots, x_{n}$. The protocol is executed in the following four phases:

\footnotetext{
${ }^{5}$ In the way it is used each prover also acts as an independent verifier.
} 
1. Each prover $P_{i}$ independently executes the algorithms

$$
\left(\operatorname{comm}_{i}, \text { state }_{i}\right) \leftarrow \operatorname{Comm}\left(x_{i}, w_{i}\right)
$$

and sends $\left(\mathrm{comm}_{i}\right)$ to the verifier.

2. The verifier selects a challenge value $c \in \mathcal{C}$ and sends it to each prover.

3. Each prover $P_{i}$, again independently, runs the algorithm

$$
\operatorname{resp}_{i} \leftarrow \operatorname{Resp}_{\left(\text {state }_{i}, c\right)}
$$

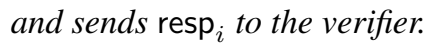

4. The verifier accepts if $\operatorname{Verify}\left(\left\{\operatorname{comm}_{i}, \operatorname{resp}_{i}, x_{i}\right\}_{i \in[n]}, c\right)=$ true.

Such a protocol should satisfy the following three properties

\section{- Correctness:}

If all $P_{i}$, each on input $\left(x_{i}, w_{i}\right) \leftarrow \mathrm{Samp}_{n}(i)$ honestly follow the protocol, then an honest verifier will accept with probability one.

- Computational Knowledge Soundness: Let $\mathcal{A}=\left(\mathcal{A}_{1}, \mathcal{A}_{2}\right)$ be a pair of PPT algorithms and $\epsilon \in[0,1)$. Consider the following game:

1. $\mathcal{A}_{1}$ is run and outputs $I \subseteq[n],\left\{x_{i}\right\}_{i \in I}$ and state $_{1}^{\mathcal{A}}$.

2. Choose $\left(x_{j}, w_{j}\right) \leftarrow \operatorname{Samp}_{n}(j)$ honestly for each $P_{j}, j \notin I$.

3. Compute $\left(\mathrm{comm}_{j}\right.$, state $\left._{j}\right) \leftarrow \operatorname{Comm}\left(x_{j}, w_{j}\right)$ for $j \notin I$.

4. $\mathcal{A}_{2}$ on input of state $_{1}^{\mathcal{A}},\left\{x_{j}, \mathrm{comm}_{j}\right\}_{j \notin I}$ outputs state $_{2}^{\mathcal{A}}$ and $\left\{\operatorname{comm}_{i}\right\}_{i \in I}$.

5. Choose $c \in \mathcal{C}$ uniformly at random and compute $\operatorname{resp}_{j} \leftarrow \operatorname{Resp}\left(\right.$ state $\left._{j}, c\right)$ for $j \notin I$.

6. $\mathcal{A}_{2}$ on input state ${ }_{2}^{\mathcal{A}}, c,\left\{\operatorname{resp}_{j}\right\}_{j \notin I}$ outputs $\left\{\operatorname{resp}_{i}\right\}_{i \in I}$.

7. We say that $\mathcal{A}_{1}, \mathcal{A}_{2}$ wins if $\operatorname{Verify}\left(\left\{\operatorname{comm}_{i}, \operatorname{resp}_{i}, x_{i}\right\}_{i \in[n]}, c\right)$ outputs true.

Assume that $\mathcal{A}$ wins the above game with probability $\delta>\epsilon$ where the probability is taken over the randomness of $\mathcal{A}_{2}$ and the choice of $c$.

Then we say that the protocol is a computational proof of knowledge if there exists a PPT algorithm Extract which, for any fixed $I,\left\{x_{i}\right\}_{i \in I}$ generated by $\mathcal{A}_{1}$, with honestly generated $\left\{x_{j}, w_{j}, \text { state }_{j}, \mathrm{comm}_{j}\right\}_{j \notin I}$ as input and black-box access to $\mathcal{A}_{2}\left(\right.$ state $\left._{2}^{\mathcal{A}},\left\{\operatorname{comm}_{j}, x_{j}\right\}_{j \notin I}\right)$ outputs $\left\{w_{i}\right\}_{i \in I}$ such that $\mathbf{P}^{\prime}\left(x_{1}, \ldots, x_{n}, w_{1}, \ldots, w_{n}\right)=1$ in expected $q($ Snd_sec $) /(\delta-\epsilon)$ steps where $q(\cdot)$ is a positive polynomial.

- Honest Verifier Zero-Knowledge: There exists a PPT algorithm $\operatorname{Sim}_{I}$ indexed by a set $I \subset[n]$, which takes as input an element in the language $\mathbb{L}$ and a challenge $c \in \mathcal{C}$, and outputs tuples $\left\{\operatorname{comm}_{i}, \operatorname{resp}_{i}\right\}_{i \notin I}$. We require that for all such I the output of $\operatorname{Sim}_{I}$ is statistically indistinguishable from a valid execution of the protocol.

Since the execution of the commitment and response phases are independent for each player, we only need to look at indistinguishability of the distribution of the values $\left(c,\left\{\operatorname{comm}_{i}, \operatorname{resp}_{i}\right\}_{i \notin I}\right)$ produced in a valid and a simulated execution of the protocol. Whatever the adversary does cannot affect the zero-knowledge property, as the values sent by the honest provers are generated independently of those sent by adversarially-controlled parties. Our formalism for HV-ZK is therefore only to allow the simulator to be applied when some provers are honest.

The knowledge extraction follows the standard definition of a proof of knowledge, but also incorporates the slackdefinition in [3] adapted to our situation of $n$-Prover ZKPoK-protocols: Note that we are not assuming a special soundness definition as is usual in $\Sigma$-protocols, since we allow the knowledge extractor to perform multiple rewind queries to the dishonest provers with correlated challenges. Also note that the knowledge extractor above outputs a witness for a predicate $\mathbf{P}^{\prime}$ which is potentially different from the predicate that honest parties were using. In traditional ZK proofs-protocols we have that $\mathbf{P}=\mathbf{P}^{\prime}$ but in many lattice based protocols these two predicates are distinct.

The above definitions imply two security parameters Snd_sec and ZK_sec. The soundness parameter Snd_sec con-

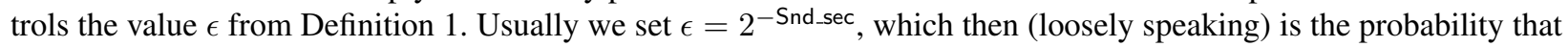
an adversary with control over a set of provers $I \subseteq[n]$ can make an honest verifier accept for values $\left\{x_{i}\right\}_{i \in I}$ without actually having valid witnesses $w_{i}$. The second parameter is the zero-knowledge parameter ZK_sec, which defines the statistical distance between the distributions of genuine and simulated transcripts. We let this distance be bounded by $2^{- \text {ZK_sec }}$. 
Relations to Other Definitions. It might seem that the above definition is related to multi-prover interactive proofs [6], but this is not true. Firstly, the provers in our definition may arbitrarily collude during the above protocol which differs from multi-prover proofs where they cannot coordinate. Moreover, our definition can be seen as each $P_{i}$ individually trying to convince the verifier about the correctness of an individual statement, but the soundness takes into account a combination of the individual statements as expressed by the language $\mathbb{L}$. Therefore, in an $n$-party ZK proof of knowledge it might happen that any subset of $n-1$ successful provers cannot produce a correct witness for the overall statement $\left(x_{1}, \ldots, x_{n}\right)$ by pooling their $w_{i}$. In multi-prover interactive proofs, each $P_{i}$ may itself have full knowledge of the complete witness.

Definition 1 also differs from just running $n$ proofs in parallel, as the predicate $\mathbf{P}$ might introduce constraints over all $x_{i}, w_{i}$. This is exactly how [25] used it in their work, where they perform checks both on the individual ciphertexts and on all of them simultaneously, thus saving runtime.

\section{A $n$-Prover ZKPoK for SPDZ}

Our protocol, which we call TopGear, is given in Figure 1 . The protocol is a $n$-Prover ZKPoK-Protocol in which, in the way we have described it, the $n$ players act both as a set of provers and individually as verifiers; as this is how the proof will be used in the SPDZ offline phase. In our description in Figure 1 the challenge of the ZKPoK is produced via calling a random functionality $\mathcal{F}_{\text {Rand }}$ which produces a single joint challenge between the players. Such a functionality is standard, see for example [15].

Recall from [15] that the proof is used in two ways. In the standard way, where flag $=\perp$ there is no extra condition on the plaintext, however when flag = Diag the underlying plaintexts are required to be the constant polynomial. To understand its workings and security, first we give the two languages and then a security proof for the standard case flag $=\perp$. The reason for this flag is that in the SPDZ protocol [15], at one stage ciphertexts need to be proved to be "Diagonal", namely each plaintext slot component contains the same element. We will discuss this after giving the proof for the main protocol.

The Honest Language: In an honest execution of the preprocessing each party $P_{i}$ first generates a set of $U$ ciphertexts given by, for $k \in[U]$,

$$
\mathfrak{c t}_{i}^{(k)}=\operatorname{Enc}\left(m_{i}^{(k)},\left(r_{i}^{(k, 1)}, r_{i}^{(k, 2)}, r_{i}^{(k, 3)}\right) ; \mathfrak{p k}\right) .
$$

Party $P_{i}$ wishes to keep the values $m_{i}^{(k)}, r_{i}^{(k, 1)}, r_{i}^{(k, 2)}, r_{i}^{(k, 3)}$ private, whereas the ciphertexts $\mathfrak{c t}_{i}^{(k)}$ are public. If we define $C_{i}=\left(\mathfrak{c t}_{i}^{(1)}, \ldots, \mathfrak{c t}_{i}^{(U)}\right)$ and $\boldsymbol{m}_{i}, R_{i}$ equivalently, then we can instead say that $P_{i}$ wishes to prove knowledge of $w_{i}=\left(\boldsymbol{m}_{i}, R_{i}\right)$ for a given $x_{i}=\left(C_{i}\right)$. By abuse of notation we relate to $\boldsymbol{m}_{i}, R_{i}, C_{i}$ via the equation $C_{i} \leftarrow$ $\operatorname{Enc}\left(\boldsymbol{m}_{i}, R_{i} ; \mathfrak{p k}\right)$.

The proof shows a statement about $\boldsymbol{m}=\sum_{i=1}^{n} \boldsymbol{m}_{i}, R=\sum_{i=1}^{n} R_{i}$ and $C=\sum_{i=1}^{n} C_{i}$, i.e. when summed over all parties. It works over $\mathcal{R}$ (and not $\mathcal{R}_{q_{1}}$ ) to make $\|\cdot\|_{\infty}$ meaningful.

We define the "honest" language $\mathbb{L}$ as

$$
\begin{aligned}
\mathbb{L}=\left\{\left(\left(x_{1}, \ldots, x_{n}\right),\right.\right. & \left.\left(w_{1}, \ldots, w_{n}\right)\right): \\
& x_{i}=C_{i}, \quad w_{i}=\left(\boldsymbol{m}_{i}, R_{i}\right), \\
& C=\sum C_{i}, \quad \boldsymbol{m}=\sum \boldsymbol{m}_{i}, \quad R=\sum R_{i}, \\
& C=\operatorname{Enc}(\boldsymbol{m}, R ; \mathfrak{p k}) \text { and for all } k \in[U] \\
& \left.\left\|\boldsymbol{m}^{(k)}\right\|_{\infty} \leq n \cdot p / 2, \quad\left\|R^{(k, \ell)}\right\|_{\infty} \leq n \cdot \rho_{\ell}\right\} .
\end{aligned}
$$

where $\rho_{1}=\rho_{2}=20$ and $\rho_{3}=1$. Note, the language says nothing about whether the initial witnesses encrypt to the initial public values $C_{i}$, it only considers a joint statement about all players' inputs. In the above definition we abuse notation by using Enc as a procedure irrespective of the distributions of the input variables (as $\boldsymbol{m}^{(k)}$ and $R^{(k, \ell)}$ are now elements in $\mathcal{R}$ and not necessarily in the correct domain). Here we simply apply the equations

$$
b \cdot R^{(k, 3)}+p \cdot R^{(k, 1)}+\boldsymbol{m}^{(k)}\left(\bmod q_{1}\right), \quad a \cdot R^{(k, 3)}+p \cdot R^{(k, 2)} \quad\left(\bmod q_{1}\right) .
$$




\section{Protocol $\Pi_{\mathrm{ZKPoK}}$}

The protocol is parametrized by integer parameters $U, V$ and flag $\in\{\operatorname{Diag}, \perp\}$ as well as $\mathfrak{p k}$ and further parameters of the encryption scheme.

\section{Sampling Algorithm: Samp}

1. If flag $=\perp$ then generate the plaintext $\boldsymbol{m} \in \mathcal{R}_{p}^{U}$ (considered as an element of $\mathcal{R}_{q_{1}}^{U}$ ) uniformly at random in $\mathcal{R}_{p}^{U}$. If flag = Diag then instead for each $k \in[U]$ let $\boldsymbol{m}^{(k)}$ be a random "diagonal" message in $\mathcal{R}_{p}$.

2. Generate a randomness triple as $R \in \mathcal{R}_{q_{1}}^{U \times 3}$, each of whose rows is generated from $\operatorname{RC}\left(\sigma^{2}, 0.5, N\right)$.

3. Compute the ciphertexts by encrypting each row separately, thus obtaining $C \leftarrow \operatorname{Enc}(\boldsymbol{m}, R ; \mathfrak{p k}) \in \mathcal{R}_{q_{1}}^{U \times 2}$.

4. Output $(x=(C), w=(\boldsymbol{m}, R))$.

Commitment Phase: Comm

1. Each $P_{i}$ samples $V$ pseudo-plaintexts $\boldsymbol{y}_{i} \in \mathcal{R}_{q_{1}}^{V}$ and pseudo-randomness vectors $S_{i}=\left(s_{i}^{(l, \ell)}\right) \in \mathcal{R}_{q_{1}}^{V \times 3}$ such that, for all $l \in[V],\left\|\boldsymbol{y}_{i}^{(l)}\right\|_{\infty} \leq 2^{\mathrm{ZK} \_ \text {sec-1 }} \cdot p$ and $\left\|s_{i}^{(l, \ell)}\right\|_{\infty} \leq 2^{\mathrm{ZK} \_ \text {sec }} \cdot \rho_{\ell}$. If flag = Diag then each $\boldsymbol{y}_{i}$ contains the same value in each plaintext slot.

2. Party $P_{i}$ computes $A_{i} \leftarrow \operatorname{Enc}\left(\boldsymbol{y}_{i}, S_{i} ; \mathfrak{p k}\right) \in \mathcal{R}_{q_{1}}^{V \times 2}$.

3. The players broadcast comm $i \leftarrow A_{i}$.

Challenge Phase: Chall

1. Parties call $\mathcal{F}_{\text {Rand }}$ to obtain a $V \times U$ challenge matrix $W$.

2. If flag $=\perp$ this is a matrix with random entries in $\left\{X^{i}\right\}_{i=0 \ldots, 2 \cdot N-1} \cup\{0\}$. If flag $=$ Diag then $W$ is a random matrix in $\{0,1\}^{V \times U}$.

Response Phase: Resp

1. Each $P_{i}$ computes $\boldsymbol{z}_{i} \leftarrow \boldsymbol{y}_{i}+W \cdot \boldsymbol{m}_{i}$ and $T_{i} \leftarrow S_{i}+W \cdot R_{i}$.

2. Party $P_{i}$ sets resp ${ }_{i} \leftarrow\left(\boldsymbol{z}_{i}, T_{i}\right)$, and broadcasts resp .

Verification Phase: Verify

1. Each party $P_{i}$ computes $D_{i} \leftarrow \operatorname{Enc}\left(\boldsymbol{z}_{i}, T_{i} ; \mathfrak{p k}\right)$.

2. The parties compute $A \leftarrow \sum_{i=1}^{n} A_{i}, C \leftarrow \sum_{i=1}^{n} C_{i}, D \leftarrow \sum_{i=1}^{n} D_{i}, T \leftarrow \sum_{i=1}^{n} T_{i}$ and $\boldsymbol{z} \leftarrow \sum_{i=1}^{n} \boldsymbol{z}_{i}$.

3. The parties check whether $D=A+W \cdot C$, and then whether the following inequalities hold, for $l \in[V]$,

$$
\left\|\boldsymbol{z}^{(l)}\right\|_{\infty} \leq n \cdot 2^{\mathrm{ZK} \_ \text {sec }} \cdot p, \quad\left\|T^{(l, \ell)}\right\|_{\infty} \leq 2 \cdot n \cdot 2^{\mathrm{ZK} \_ \text {sec }} \cdot \rho_{\ell} \text { for } \ell=1,2,3 .
$$

4. If flag $=$ Diag then the proof is rejected if $\boldsymbol{z}^{(l)}$ is not a constant polynomial (i.e. a "diagonal" plaintext element).

5. If all checks pass, the parties accept, otherwise they reject.

Figure 1. Protocol for global proof of knowledge of a set of ciphertexts

For dishonest provers (where we assume the worst case of all provers being dishonest) we will only be able to show that the inputs are from the language

$$
\begin{aligned}
\mathbb{L}_{c}^{\prime}=\left\{\left(\left(x_{1}, \ldots, x_{n}\right),\right.\right. & \left.\left(w_{1}, \ldots, w_{n}\right)\right): \\
& x_{i}=C_{i}, \quad w_{i}=\left(\boldsymbol{m}_{i}, R_{i}\right), \\
& C=\sum C_{i}, \quad \boldsymbol{m}=\sum \boldsymbol{m}_{i}, \quad R=\sum R_{i}, \\
& C=\operatorname{Enc}(\boldsymbol{m}, R ; \mathfrak{p k}) \text { and for all } k \in[U] \\
& \left\|c \cdot \boldsymbol{m}^{(k)}\right\|_{\infty} \leq 2^{\mathrm{ZK} \_ \text {sec }+1} \cdot n \cdot p, \\
& \left.\left\|c \cdot R^{(k, \ell)}\right\|_{\infty} \leq 2^{\mathrm{ZK} \text { sec }+2} \cdot n \cdot \rho_{\ell}\right\} .
\end{aligned}
$$


Notice that not only the bounds on $\boldsymbol{m}^{(k)}, R^{(k)}$ have increased, but the values whose norms are determined have also been multiplied by a factor of $c$.

Thus we see that, at a high level, the bounds for the honest language are $\left|w_{i}\right|<B$, whilst the bounds for the proven language are $\left|c \cdot w_{i}\right|<2^{\mathrm{ZK} \_s e c+2} \cdot B$. This additional factor $2^{\mathrm{ZK} \_s e c+2}$ is called the soundness slack. This soundness slack could be reduced by utilizing rejection sampling as in lattice signature schemes, but this would complicate the protocol (being an $n$-party proof), lead to a slowdown due to having to potentially rerun the protocol multiple times and (more importantly) it turns out that the soundness slack has no important effect on the parameters needed in practice.

There is an added complication arising from the language $\mathbb{L}_{c}^{\prime}$, corresponding to the factor of $c$ in Lemma 2 However, this can be side-stepped by a minor modification to how the ZKPoKs are used with the SPDZ offline phase (which we describe in Section 5 .

Theorem 1. Let flag $=\perp$ and $V \geq($ Snd_sec +2$) / \log _{2}(2 N+1)$, then the algorithms in Figure 1 are an n-party $Z K P o K$ protocol according to Definition 1 for the languages $\mathbb{L}$ and $\mathbb{L}_{2}^{\prime}$ with soundness error $2^{- \text {Snd_sec }}$ and statistical distance $2^{-\mathrm{ZK} \_ \text {sec }}$ in the simulation.

Proof.

Correctness: In proving the correctness property all the parties are assumed to be honest. We therefore have that $\left(x_{i}, w_{i}\right) \leftarrow \operatorname{Samp}_{n}(i)$ are generated by the sampling algorithm. Thus for all $k \in[U],\left\|\boldsymbol{m}_{i}^{(k)}\right\|_{\infty} \leq \frac{p}{2}$ and $\left\|r_{i}^{(k, \ell)}\right\|_{\infty} \leq$ $\rho_{\ell}$, where $\rho_{1}=\rho_{2}=20$ and $\rho_{3}=1$. As summing over the $n$ values increases the overall norm by at most $n$, we have $\left(\left(x_{1}, \ldots, x_{n}\right),\left(w_{1}, \ldots, w_{n}\right)\right) \in \mathbb{L}$.

We now prove that the protocol terminates by considering the checks in Step 3 of Verify. The equality $D=$ $A+W \cdot C$ follows at once from the fact that everything is defined as a linear function of their arguments and the BGV encryption, in particular, is linear and works component-wise. By repeatedly applying Lemma 3 (and using the fact that $U \leq 2^{\text {ZK_sec }}$ in practice) we obtain

$$
\begin{aligned}
\left\|\boldsymbol{z}^{(k)}\right\|_{\infty} & \leq \sum_{i=1}^{n}\left\|\left(\boldsymbol{y}_{i}+W \cdot \boldsymbol{m}_{i}\right)^{(k)}\right\|_{\infty}<n \cdot\left(2^{\mathrm{ZK} \_ \text {sec }} \cdot \frac{p}{2}+U \cdot \frac{p}{2}\right) \\
& \leq p \cdot n \cdot 2^{\mathrm{ZK} \_ \text {sec }}, \\
\left\|T^{(k, \ell)}\right\|_{\infty} & \leq \sum_{i=1}^{n}\left\|\left(S_{i}+W \cdot R_{i}\right)^{(k, \ell)}\right\|_{\infty}<n \cdot\left(2^{\mathrm{ZK} \_ \text {sec }} \cdot \rho_{\ell}+U \cdot \rho_{\ell}\right) \\
& \leq 2 \cdot n \cdot 2^{\mathrm{ZK} \_ \text {sec }} \cdot \rho_{\ell} .
\end{aligned}
$$

Honest verifier zero-knowledge: Following our definition of $n$-prover ZKPoK-protocol we give in Figure 2$]$ a simulator, parametrized by a set of corrupted parties $I$ and a challenge $W$, which produces transcripts. It is clear that the statistical difference in the distribution of the coefficients of the ring elements in $z_{j}$ and $T_{j}$ for $j \notin I$ in a real execution and the simulated execution can be bounded by Lemma 1$]$ as $2^{- \text {ZK_sec }}$.

\section{The Simulator $\operatorname{Sim}_{I}$}

The input is a challenge $V \times U$ matrix $W$ defined as in the main protocol.

1. For all $j \notin I$ do

(a) Sample $\boldsymbol{z}_{j} \in \mathcal{R}_{q_{1}}^{V}$ such that, for $l \in[V]$, we have $\left\|\boldsymbol{z}_{j}^{(l)}\right\|_{\infty} \leq 2^{\mathrm{ZK} \text {-sec-1 }} \cdot p$.

(b) Sample $T_{j} \in \mathcal{R}_{q_{1}}^{V \times 3}$ such that, for $l \in[V]$ and $\ell=1,2,3$, we have $\left\|T_{j}^{(l, \ell)}\right\|_{\infty} \leq 2^{\text {ZK_sec }} \cdot \rho_{\ell}$.

(c) Set $\operatorname{resp}_{j} \leftarrow\left(\boldsymbol{z}_{j}, T_{j}\right)$.

(d) Compute $\operatorname{comm}_{j}=A_{j} \leftarrow \operatorname{Enc}\left(\boldsymbol{z}_{j}, T_{j} ; \mathfrak{p k}\right)-W \cdot C_{j}$.

2. Output $\left(\mathrm{comm}_{j}, \operatorname{resp}_{j}\right)_{j \notin I}$.

Figure 2. Simulator $\operatorname{Sim}_{I}$ for our protocol

Knowledge soundness: The knowledge extractor follows the methodology of [3, Lemma 3 and 5]. We refer the reader there for more details. Here we outline the technique and the effect it has on our bounds for the language $\mathbb{L}_{2}^{\prime}$. 
In the following we let $\delta$ denote the probability that, for fixed $I,\left\{x_{i}\right\}_{i \in I}$ of dishonest provers $\mathcal{A}_{2}$ produces a valid transcript (sampled over the randomness tape $\chi$ of $\mathcal{A}_{2}$, the possible challenges $W$ and the values $\left\{A_{j}, \boldsymbol{z}_{j}, T_{j}\right\}_{j \notin I}$ ). By assumption we have $\delta>2^{- \text {Snd_sec }}$.

We first need to ensure that we can apply the proof of [3] in our setting. Their construction only has a single prover to extract from and they do not have extra messages which honest participants in the protocol may send. To show applicability of their extractor, we construct a "hybrid" prover $\hat{\mathcal{P}}$ that can be combined with their technique.

Claim: Let $\mathcal{A}$ be an adversary as in the soundness definition of Definition 1 . Then there exists a prover $\hat{\mathcal{P}}$ which as input only obtains the random tape $\hat{\chi}$ (as well as implicitly the parameters of the encryption scheme, $\left\{x_{j}, w_{j}\right\}_{j \notin I}$ generated by Samp as well as $I$ ) together with state ${ }_{1}^{\mathcal{A}}$ and a challenge $c$. The player $\hat{\mathcal{P}}$ has the same chance $\delta$ of outputting a correct transcript (when the probability is taken over $\hat{\chi}, W$ ) as $\mathcal{A}$.

Proof. We construct $\hat{\mathcal{P}}$ as follows:

1. Let $\hat{\chi}=\left(\chi_{1}|| \chi_{2}\right)$ where $\chi_{1}$ is of the same length as the randomness of $\mathcal{A}_{2}$ and $\chi_{2}$ is sufficiently long to run $n-|I|$ independent instances of Comm.

2. Compute $\left(\right.$ comm $_{j}$, state $\left.j\right) \leftarrow \operatorname{Comm}\left(x_{j}, w_{j}\right)$ for all $j \notin I$ using a fresh part of $\chi_{2}$ for each instance.

3. Run $\mathcal{A}_{2}$ on $\left\{x_{j}, \operatorname{comm}_{j}\right\}_{j \notin I}$, state ${ }_{1}^{\mathcal{A}}$, to obtain $\left\{\operatorname{comm}_{i}\right\}_{i \in I}$ and state ${ }_{2}^{\mathcal{A}}$.

4. Output $\left\{\operatorname{comm}_{i}\right\}_{i \in[n]}$ and wait for the challenge $c$.

5. Upon input $c$ compute $\left(\operatorname{resp}_{j}\right) \leftarrow \operatorname{Resp}\left(\operatorname{state}_{j}, c\right)$ for all $j \notin I$.

6. Continue to run $\mathcal{A}_{2}$ on inputs $c,\left\{\operatorname{resp}_{j}\right\}_{j \notin I}$, state ${ }_{2}^{\mathcal{A}}$. Then output $\left\{\operatorname{resp}_{j}\right\}_{j \notin I}$ and whatever $\mathcal{A}_{2}$ outputs.

Observe that by assumption Resp is a deterministic algorithm. Therefore, there is only one value $\left\{\operatorname{resp}_{j}\right\}_{j \notin I}$ which we can give to $\mathcal{A}_{2}$ in Step 6 of the soundness game. Thus, $\mathcal{A}_{2}$ 's actions are fully determined given $\chi, c,\left\{x_{j}, \operatorname{comm}_{j}\right\}_{j \notin I}$, state $_{1}^{\mathcal{A}}$. As $\hat{\mathcal{P}}$ generates $\left\{\mathrm{comm}_{j}\right\}$ in the same way as it is done in the security experiment, it has the same chance $\delta$ of outputting a correct transcript (when probability is taken over $\hat{\chi}, c$ ) as $\mathcal{A}$. The only additional runtime comes from running Comm, Resp for simulated honest parties, which is negligible.

They then construct an ensemble of extractors $\left\{\hat{\mathcal{E}}_{k}\right\}_{k \in[U]}$, one for each of the inputs. This also works in our setting, as we can extract each $k$-th plaintext and randomness from each of the parties simultaneously. More in detail, each $\hat{\mathcal{E}}_{k}$, when adapted to our setting, works as follows:

1. Run $\hat{\mathcal{P}}$ on random challenges $W$ and uniformly random choices of $\hat{\chi}$ until it outputs an accepting transcript. Save the accepting challenge $W$ as well as response $\left\{\boldsymbol{z}_{i}, T_{i}\right\}_{i=1}^{n}$.

2. Select a new challenge matrix $W^{(k)}$ which is identical to $W$ except in column $k$. This challenge is passed to $\hat{\mathcal{P}}(\hat{\chi})$ and the process is repeated, either until one of the $W^{(k)}$ succeeds or if Snd_sec/ $\delta$ challenges were generated but none succeeded. In the latter case, the extractor aborts.

3. If the extractor succeeds, then it outputs $\left\{\boldsymbol{z}_{i}, \boldsymbol{z}_{i}^{(k)}, T_{i}, T_{i}^{(k)}\right\}_{i \in[n]}$ as well as $W, W^{(k)}$.

Assume that $\hat{\mathcal{E}}_{k}$ succeeds in outputting the two accepting transcripts $W,\left\{\boldsymbol{z}_{i}, T_{i}\right\}_{i=1}^{n}, W^{(k)},\left\{\boldsymbol{z}_{i}^{(k)}, T_{i}^{(k)}\right\}_{i \in[n]}$ with the constraints as given above. Then, for this $k \in[U]$ we can compute the following: First, let $l \in[V]$ denote an index such that $w_{l, k} \neq w_{l, k}^{\prime}$, where $w_{i, j}$ (resp. $w_{i, j}^{\prime}$ ) are the entries of $W$ (resp. $W^{(k)}$ ). Using Lemma 2 we can write $g=1 /\left(w_{l, k}-w_{l, k}^{\prime}\right) \in \mathcal{R}$ with $\|2 \cdot g\|_{\infty} \leq 1$.

Setting $D_{i} \leftarrow \operatorname{Enc}\left(\boldsymbol{z}_{i}, T_{i} ; \mathfrak{p k}\right)$ and $D_{i}^{(k)} \leftarrow \operatorname{Enc}\left(\boldsymbol{z}_{i}^{(k)}, T_{i}^{(k)} ; \mathfrak{p k}\right)$ we obtain two matrix equations

$$
D=A+W \cdot C \text { and } D^{(k)}=A+W^{(k)} \cdot C .
$$

Write $E=D-D^{(k)}$ and note that the $(i, j)^{\prime}$ 'th element $e_{i, j}$ is equal to

$$
e_{i, j}=\sum_{t=1}^{U}\left(w_{i, t}-w_{i, t}^{\prime}\right) \cdot c_{t, j}=\left(w_{i, k}-w_{i, k}^{\prime}\right) \cdot c_{k, j},
$$

by choice of $W^{(k)}$. Hence $2 \cdot c_{k, j}=2 \cdot g \cdot e_{l, j}$, which implies, by the linearity of encryption, that we can extract a message $m^{(k)}$ and randomness values corresponding to row $R^{(k, \cdot)}$, which satisfy the bounds for $\ell=1,2,3$

$$
\left\|2 \cdot m^{(k)}\right\|_{\infty} \leq 2 \cdot n \cdot 2^{\mathrm{ZK} \_ \text {sec }+1} \cdot \frac{p}{2}
$$




$$
\left\|2 \cdot R^{(k, \ell)}\right\|_{\infty} \leq 2 \cdot n \cdot 2^{\mathrm{ZK} \_ \text {sec }+1} \cdot \rho_{\ell} .
$$

In a similar way as above we can moreover extract the individual $m_{i}^{(k)}, R_{i}^{(k)}$ which add up to $m^{(k)}, R^{(k)}$. By concatenation of the outputs which we obtain this way from all $\hat{\mathcal{E}}_{k}$, this then permits to output a witness for $\mathbb{L}_{2}^{\prime}$ as required.

We now consider runtime and success probability of this process. Following the analysis of [3, Lemma 5], Step 1 of $\hat{\mathcal{E}}_{k}$ takes expected time $1 / \delta$ to succeed. Afterwards in Step 2 of each $\hat{\mathcal{E}}_{k}$ we make at most Snd_sec/ $\delta$ queries to $\mathcal{P}$. Following the analysis of [3], by running $\hat{\mathcal{E}}_{k}$ at most 3 . Snd_sec times it will output a pair of values, except with probability at most $2^{- \text {Snd_sec }}$. By a union bound, all the above extractors will output a witness for $\mathbb{L}_{2}^{\prime}$ in expectancy ${ }^{6}$ in time $\left(3 U \cdot\right.$ Snd_sec $\left.^{2}\right) / \delta$ with probability at least $1-U \cdot 2^{\text {Snd_sec }}$.

The value $q(\lambda, U)=3 \cdot U \cdot$ Snd_sec $^{2}$ in the above proof should, correctly, be taken into account in our security estimates later. However, the effect is marginal, and so to aid exposition we drop this consideration from now on.

For the ZKPoK of "diagonal" elements, the main difference is that soundness must ensure that the extracted $\boldsymbol{m}$ encodes a "diagonal" plaintext as well. Unfortunately, as we have to multiply with ring elements in the extraction process of the soundness argument, it cannot be guaranteed that the outcome is "diagonal". Instead, in such a case we fall back to a binary challenge matrix $W$ (as depicted in Figure 11, where the "diagonal" property follows as the extractor only performs additions and subtractions on the values $\boldsymbol{z}_{i}, T_{i}$ in the process, but no multiplications with inverses. As a side effect, the proof actually yields bounds on $\boldsymbol{m}^{(k)}, R^{(k, \ell)}$ for $c=1$. One can easily show the following

Corollary 1. Let flag = Diag and $V \geq$ Snd_sec +2 , then the algorithms in Figure 1 lare an n-party ZKPoK protocol according to Definition 1 for the languages $\mathbb{L}$ and $\mathbb{L}_{1}^{\prime}$ with soundness error $2^{- \text {Snd_sec }}$ and statistical distance $2^{-Z K_{-} s e c}$ in the simulation.

\section{SPDZ Offline Phase}

We now show how to combine the ZKPoK from Section 4 with the offline phase of the SPDZ protocol. After a brief recap of it, we outline the necessary changes to the HighGear protocol of [25]. Recall that the offline phase of SPDZ primarily generates shared random triples $(\langle a\rangle,\langle b\rangle,\langle c\rangle)$ such that $c=a \cdot b$ where $a, b$ are chosen uniformly at random from $\mathbb{F}_{p}$ (and no subset of parties either know $a, b$ or $c$, or can affect their distribution). This is done, by each party $P_{i}$ encoding $\phi(m) \boldsymbol{a}_{i}$ and $\boldsymbol{b}_{i}$ values into two elements $a_{i}$ and $b_{i}$ in $\mathcal{R}_{p}$. These $a_{i}$ and $b_{i}$ are encrypted via the BGV scheme, and the parties obtain $\mathfrak{c t}_{a_{i}}=\operatorname{Enc}\left(a_{i}, \boldsymbol{r}_{a, i} ; \mathfrak{p k}\right)$ and $\mathfrak{c t}_{b_{i}}=\operatorname{Enc}\left(b_{i}, \boldsymbol{r}_{b, i} ; \mathfrak{p k}\right)$.

Using the homomorphic properties of the BGV encryption scheme the parties can then compute an encryption of the product $c \in \mathcal{R}_{p}$ via

$$
\mathfrak{c t}_{c}=\left(\mathfrak{c t}_{a_{1}} \boxplus \cdots \boxplus \mathfrak{c t}_{a_{n}}\right) \odot\left(\mathfrak{c t}_{b_{1}} \boxplus \cdots \boxplus \mathfrak{c t}_{b_{n}}\right) .
$$

The product $\mathfrak{c t}_{c}$ is decrypted using a distributed decryption protocol which gives to each party a share $c_{i} \in \mathbb{F}_{p}$ of the plaintext of $\mathfrak{c t}_{c}$. To achieve security in the online phase, one furthermore needs to compute shares of the MACs $\gamma[a], \gamma[b]$ and $\gamma[c]$, which are obtained in a similar manner. In order to enforce input independence, the parties do not merely exchange $\mathfrak{c t}_{a_{i}}, \mathfrak{c t}_{b_{i}}$ but instead first commit to these ciphertexts before revealing them afterwards.

In the offline phase, the main attack vector is that dishonest parties could produce ciphertexts which contain maliciously chosen noise or a plaintext unbeknownst to the sending party. This would result in either selective failure attacks or information leakage during the distributed decryption procedure. Thus each ciphertext $\mathfrak{c t}_{a_{i}}$ needs to be accompanied by a ZKPoK showing that it is not too far from being an honestly generated ciphertext. As the ZKPoKs bound the noise term associated to every ciphertext, we use this bound to derive the parameters for the BGV encryption scheme. This in turn ensures that all ciphertexts will validly decrypt. Quite obviously we want to execute $U$ such proofs in parallel such as to amortize.

As noticed in HighGear [25], the ciphertexts $\mathfrak{c t}_{a_{i}}$ are only ever used in a sum (as in Equation 11). Therefore it is possible to replace $n$ individual ZKPoKs for $\mathfrak{c t}_{a_{i}}$ by a single ZKPoK for the sum $\mathfrak{c t}_{a}=\mathfrak{c t}_{a_{1}} \boxplus \cdots \boxplus \mathfrak{c t}_{a_{n}}-$ which is exactly the strategy we outlined in the previous two sections. However, our proof comes at the expense of not

\footnotetext{
${ }^{6}$ One can improve the runtime by making a different analysis: as the success probability for each run of $\hat{\mathcal{E}_{k}}$ is constant, one can analyze the chance of $O(\lambda)$ consecutive calls to different extractors having $\geq U$ success events using a Hoeffding bound.
} 
obtaining guarantees about the original ciphertext sum $\mathfrak{c t}_{a}$, but instead of $2 \cdot \mathfrak{c t}_{a}=\mathfrak{c t}_{a} \boxplus \mathfrak{c t}_{a}$. Luckily this of no concern in preprocessing for SPDZ - we can simply later adjust some of the shares by a factor of two and continue as before. The modifications are explained in Figure 3 for the case of triple production. The modifications to obtain other forms of preprocessed data such as those in [14] are immediate.

\section{Protocol $\Pi_{\text {offline }}$}

Init:

1. Each $P_{i}$ locally runs $\operatorname{Samp}_{n}(i)$ with flag $=$ Diag and $U=1$ to obtain $\alpha_{i} \in \mathbb{F}_{p}$ as well as the ciphertext $\mathfrak{c t}_{\alpha_{i}}$.

2. Each $P_{i}$ broadcasts a commitment to $\mathfrak{c t}_{\alpha_{i}}$. Upon receiving all such commitments, each party broadcasts the opening to the commitment. If any such opening fails, then abort.

3. For $i=1, \ldots$, Snd_sec $/ 16$

(a) The parties perform the other phases of $\Pi_{\text {ZKPoK }}$ with flag $=\operatorname{Diag}, U=1$ and $V=16$. If any proof rejects they abort.

4. The parties set $\mathfrak{c t}_{\alpha} \leftarrow\left(\mathfrak{c t}_{\alpha_{1}} \boxplus \cdots \boxplus \mathfrak{c t}_{\alpha_{n}}\right)$.

\section{Triples:}

1. We set $V=($ Snd_sec +2$) / \log _{2}(2 \cdot N+1)$ and $U=2 \cdot V$.

2. Each $P_{i}$ runs $\operatorname{Samp}_{n}(i)$ for this value of $U$ with flag $=\perp$. It thus obtains the plaintext vectors $\hat{\boldsymbol{a}}_{i}^{(k)}, \hat{\boldsymbol{b}}_{i}^{(k)}, \hat{\boldsymbol{f}}_{i}^{(k)} \in\left(\mathbb{F}_{p}\right)^{\phi(m)}$ as well as the ciphertexts $\mathfrak{c t}_{\hat{a}_{i}}^{(k)}, \mathfrak{c t}_{\hat{b}_{i}}^{(k)}$ and $\mathfrak{c t}_{\hat{f}_{i}}^{(k)}$ for $k \in[U]$.

3. Each $P_{i}$ broadcasts commitments to $\mathfrak{c t}_{\hat{a}_{i}}^{(k)}, \mathfrak{c t}_{\hat{b}_{i}}^{(k)}$ and $\mathfrak{c t}_{\hat{f}_{i}}^{(k)}$ for $k \in[U]$. Upon receiving all such commitments, each party broadcasts the opening to the commitments. If any such opening fails, then abort.

4. The parties then run the remaining steps of the protocol $\Pi_{\text {ZKPoK }}$ using $U, V$ and flag $=\perp$. If any of the proofs fail, then they abort.

5. The parties set $\mathfrak{c t}_{a}^{(k)} \leftarrow 2 \cdot\left(\mathfrak{c t}_{\hat{a}_{1}}^{(k)} \boxplus \cdots \boxplus \mathfrak{c t}_{\hat{a}_{n}}^{(k)}\right), \mathfrak{c t}_{b}^{(k)} \leftarrow 2 \cdot\left(\mathfrak{c t}_{\hat{b}_{1}}^{(k)} \boxplus \cdots \boxplus \mathfrak{c t}_{\hat{b}_{n}}^{(k)}\right)$ and $\mathfrak{c t}_{f}^{(k)} \leftarrow 2 \cdot\left(\mathfrak{c t}_{\hat{f}_{1}}^{(k)} \boxplus \cdots \boxplus \mathfrak{c t}_{\hat{f}_{n}}^{(k)}\right)$ for $k \in[U]$.

6. The parties compute $\mathfrak{c t}_{c}^{(k)} \leftarrow \mathfrak{c t}_{a}^{(k)} \odot \mathfrak{c t}_{b}^{(k)}$ as well as $\mathfrak{c t}_{c+f}^{(k)} \leftarrow \mathfrak{c t}_{c}^{(k)} \boxplus \mathfrak{c t}_{f}^{(k)}$ for $k \in[U]$.

7. Using the distributed decryption operation of the BGV scheme they then decrypt $\mathfrak{c t}_{c+f}^{(k)}$ for $k \in[U]$, to obtain $\boldsymbol{\Delta}^{(k)}$.

8. $P_{1}$ sets $\hat{\boldsymbol{c}}_{1}^{(k)} \leftarrow \boldsymbol{\Delta}^{(k)}-\boldsymbol{f}_{1}^{(k)}$, while each remaining $P_{i}$ sets $\hat{\boldsymbol{c}}_{i}^{(k)} \leftarrow-\boldsymbol{f}_{i}^{(k)}$ for $k \in[U]$.

9. The parties compute a fresh encryption of each $\hat{\boldsymbol{c}}^{(k)}$ via $\tilde{\mathfrak{c t}}_{c}^{(k)} \leftarrow \operatorname{Enc}\left(\boldsymbol{\Delta}^{(k)}, \mathbf{0} ; \mathfrak{p k}\right)-\mathfrak{c t}_{f}^{(k)}$ with default random coins $\mathbf{0}$ for $k \in[U]$.

10. The parties compute $\mathfrak{c t}_{\alpha \cdot a}^{(k)} \leftarrow \mathfrak{c t}_{\alpha} \odot \mathfrak{c t}_{a}^{(k)}, \mathfrak{c t}_{\alpha \cdot b}^{(k)} \leftarrow \mathfrak{c t}_{\alpha} \odot \mathfrak{c t}_{b}^{(k)}$ and $\mathfrak{c t}_{\alpha \cdot c}^{(k)} \leftarrow \mathfrak{c t}_{\alpha} \odot \tilde{c t}_{c}^{(k)}$ for $k \in[U]$.

11. The MAC values $\gamma_{i}^{(k)}[\boldsymbol{a}], \gamma_{i}^{(k)}[\boldsymbol{b}], \gamma_{i}^{(k)}[\boldsymbol{c}]$ are obtained by applying the DistDec protocol in Figure 14 from [25] for $k \in[U]$.

12. Each party sets $\boldsymbol{a}_{i}^{(k)} \leftarrow 2 \cdot \hat{\boldsymbol{a}}_{i}^{(k)}, \boldsymbol{b}_{i}^{(k)} \leftarrow 2 \cdot \hat{\boldsymbol{b}}_{i}^{(k)}$ and $\boldsymbol{c}_{i}^{(k)} \leftarrow 4 \cdot \hat{\boldsymbol{c}}_{i}^{(k)}$. It then obtains the shares of the elements encoded in $\boldsymbol{a}_{i}^{(k)}, \boldsymbol{b}_{i}^{(k)}, \boldsymbol{c}_{i}^{(k)}$ as well as their MACs $\gamma_{i}^{(k)}[\boldsymbol{a}], \gamma_{i}^{(k)}[\boldsymbol{b}], \gamma_{i}^{(k)}[\boldsymbol{c}]$ by mapping the associated polynomials into the slot representation. They thus obtain $U \cdot \phi(m)$ shares.

Figure 3. TopGear version of the SPDZ Offline Phase

The overhead $V$ for the ciphertexts encrypting $\alpha_{i}$ is quite big when compared to those of the triples and MAC shares. This is because our proof from Section 4 is not as efficient when flag = Diag (see Corollary 1). However, this is not an issue as we only produce one such ciphertext during the offline phase. To mitigate this we actually run the ZKPoK for a fixed value $(V=16)$ and then repeat this Snd_sec/ $V$ times. This makes no difference to the overall running time, but keeps the memory requirements low for this part of the protocol.

In the protocol in Figure 3 we have utilized the more efficient Distributed Decryption protocol from HighGear to obtain the MAC shares, and have merged in the ReShare protocol of [14, Figure 11]. This protocol is needed to obtain the shares of $c$ and the fresh encryption of $c$.

The proof of security of this offline phase follows exactly as in the original SPDZ papers [15|14], all that changes is the bound on the noise of the resulting ciphertexts. Suppose $\left(c_{0}, c_{1}\right)$ is a ciphertext corresponding to one of $\mathfrak{c t}_{\alpha}, \mathfrak{c t}_{a}, \mathfrak{c t}_{b}$ 
or $\mathfrak{c t}_{f}$ in our protocol. To prove security, it is necessary to obtain worst case bounds on the value $\left\|c_{0}-\mathfrak{s k} \cdot c_{1}\right\|_{\infty}^{\text {can }}$. We know that

$$
c_{0}-\mathfrak{s k} \cdot c_{1}=2 \cdot \sum_{i=1}^{n}\left(m_{i}+p \cdot\left(\epsilon \cdot r_{i}^{(3)}+r_{i}^{(1)}-r_{i}^{(2)} \cdot \mathfrak{s k}\right)\right)
$$

where $\left(m_{i}, r_{i}^{(1)}, r_{i}^{(2)}, r_{i}^{(3)}\right)$ are bounded due to the ZKPoK. From the soundness of it we can guarantee that the ciphertexts must satisfy

$$
\left\|2 \cdot \sum_{i \in[n]} m_{i}\right\|_{\infty} \leq 2^{\mathrm{ZK} \_s e c+1} \cdot n \cdot p \quad \text { and } \quad\left\|2 \cdot \sum_{i \in[n]} r_{i}^{(\ell)}\right\|_{\infty} \leq 2^{\mathrm{ZK} \_s e c+2} \cdot n \cdot \rho_{\ell} .
$$

Due to our assumption of an honest key generation phase, we also know that with probability $1-2^{-\epsilon}$ we have $\|\epsilon\|_{\infty}^{\text {can }} \leq \mathfrak{c}_{1} \cdot \sigma \cdot \sqrt{\phi(m)}$ and $\|\mathfrak{s k}\|_{\infty}^{\text {can }} \leq \mathfrak{c}_{1} \cdot \sqrt{h}$. Using the inequality $\|x\|_{\infty}^{\text {can }} \leq \phi(m) \cdot\|x\|_{\infty}$ we obtain

$$
\begin{aligned}
& \left\|c_{0}-\mathfrak{s k} \cdot c_{1}\right\|_{\infty}^{\mathrm{can}} \\
& \leq \sum_{i=1}^{n}\left\|2 \cdot m_{i}\right\|_{\infty}^{\mathrm{can}}+p \cdot\left(\|\epsilon\|_{\infty}^{\mathrm{can}} \cdot\left\|2 \cdot e_{2, i}\right\|_{\infty}^{\mathrm{can}}+\left\|2 \cdot e_{0, i}\right\|_{\infty}^{\mathrm{can}}+\|\mathfrak{s k}\|_{\infty}^{\mathrm{can}} \cdot\left\|2 \cdot e_{1, i}\right\|_{\infty}^{\mathrm{can}}\right) \\
& \leq 2 \cdot \phi(m) \cdot 2^{\mathrm{ZK} \_ \text {sec }+1} \cdot n \cdot p / 2 \\
& \quad+p \cdot\left(\mathfrak{c}_{1} \cdot \sigma \cdot \phi(m)^{3 / 2} \cdot 2 \cdot 2^{\mathrm{ZK} \_ \text {sec }+1} \cdot n+\phi(m) \cdot 2 \cdot 2^{\mathrm{ZK} \_ \text {sec }+1} \cdot n \cdot 20\right. \\
& \left.\quad+\mathfrak{c}_{1} \cdot \sqrt{h} \cdot \phi(m) \cdot 2 \cdot 2^{\mathrm{ZK} \_ \text {sec }+1} \cdot n \cdot 20\right) \\
& =\phi(m) \cdot 2^{\mathrm{ZK} \_ \text {sec }+2} \cdot n \cdot p \cdot\left(\frac{41}{2}+\mathfrak{c}_{1} \cdot \sigma \cdot \phi(m)^{1 / 2}+20 \cdot \mathfrak{c}_{1} \cdot \sqrt{h}\right) \\
& =B_{\text {clean }}^{\text {dishonest }} .
\end{aligned}
$$

Using this bound we can then derive the parameters for the BGV system using exactly the same methodology as can be found in [2].

\section{Results}

Recall we have three different security parameters in play, apart from the computational security parameter $\kappa$ of the underlying BGV encryption scheme. The main benefit of TopGear over HighGear is that it potentially enables higher values of the parameter Snd_sec to be obtained. Recall $2^{- \text {Snd_sec }}$ is the probability that an adversary will be able to produce a convincing ZKPoK for an invalid input. The other two security parameters are ZK_sec and DD_sec, which measure the statistical distance of coefficients of ring elements generated in a protocol to the same coefficients being generated in the simulation of the security proof.

In the context of the HighGear ZKPoK in the Overdrive paper [25] the two security parameters are set to be equal, i.e. ZK_sec = Snd_sec. In practice the value of Snd_sec needs to be very low for the HighGear ZKPoK as it has a direct effect on the memory consumption of the underlying protocol. Thus in SCALE v1.2 the default value for Snd_sec is 40. This unfortunately translates into having a high probability of an adversary being able to get away with cheating in a ZKPoK and is therefore not desirable. The first goal that our work achieves, which will be validated with experiments in this section, is that Snd_sec can be taken to be as large as is desired, whilst also obtaining an efficiency saving.

We also aim to increase the values of ZK_sec and DD_sec. These measure statistical distances of coefficients. Picking ZK_sec and DD_sec at low values potentially introduces leakage about the plaintexts or the secret keys. Hence, after demonstrating the effect of our new protocol with respect to more secure choices of Snd_sec, we then turn to examining the effect of increasing the other security parameters as well. In Table 1 in the Appendix we give various parameter sizes for the degree $N$ and moduli $q_{0}=p_{0}, q_{1}=p_{0} \cdot p_{1}$ for different plaintext space sizes $p$, and different security levels ZK_sec, Snd_sec, DD_sec and computational security parameter $\kappa$, and two parties 7 . We selected parameters for which ZK_sec, DD_sec $\leq$ Snd_sec to keep the table managable. We use the methodology described in [2]

\footnotetext{
${ }^{7}$ Similar values can be obtained for other values of $n$, we selected $n=2$ purely for illustration here, the effect of $n$ on the values is relatively minor.
} 
to derive parameter sizes for both HighGear and TopGear; this maps the computational security parameter to lattice parameters using Albrecht's too 8 In the table a row with values of $\star$ in the ZK_sec columns means that the parameter values do not change when this parameter is to set either 40 or 80 .

From the table we see that the values of ZK_sec and Snd_sec produce relatively little effect on the overall parameter sizes, especially for large values of the plaintext modulus $p$. This is because the modulus switch, within the homomorphic evaluation, squashes the noise by a factor of at least $p$. The values ZK_sec and Snd sec only blow up the

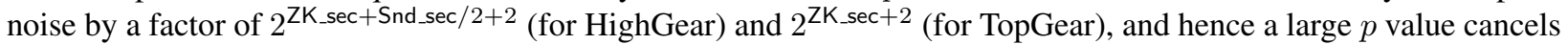
out this increase in noise due to the ZKPoK security parameters. In addition the parameter sizes are identical for both HighGear and TopGear, except in the case of some parameters for low values of $\log _{2} p$.

We based our implementation and experiments on the SCALE-MAMBA system [2] which has an implementation of the HighGear protocol. To measure the improvement due to our new TopGear protocol we modified [2] to test against the old version. We focused on the case of 128-bit plaintext moduli in our experiments, being the recommended size in SCALE-MAMBA v1.2 to support certain MPC operations such as fixed-point arithmetic. We first baselined the implementation in SCALE-MAMBA of HighGear against the implementation reported in [25]. The experiments in [25] were executed on i7-4790 and i7-3770S CPUs, compared to our experiments which utilized i7-7700K CPUs. From a pure CPU point of view our machines should be roughly $30 \%$ faster. The ping time between our machines was 0.47 milliseconds, whereas that for [25] was 0.3 milliseconds.

Keller et al [25], in the case of 128-bit plaintext moduli, and with the security settings equivalent to our setting of DD_sec $=$ ZK_sec $=$ Snd_sec $=64$, utilize a ciphertext modulus of 572 bits, whereas SCALE-MAMBA v1.2 utilizes a ciphertext modulus of 541 bits. In this setting [25] achieve a maximum throughput of 5600 triples per second, whereas SCALE-MAMBA's implementation of HighGear obtains a maximum throughput of roughly 2900 triples per second. We suspect the reason for the difference in costs is that SCALE-MAMBA is performing other operations related to storing the triples for later consumption by online operations. This also means that memory utilization grows as more triples are produced, leading to a larger amount of non-local memory accesses. These effects decrease the measurable triple production rate in SCALE-MAMBA compared to the experiments presented in [25].

We now turn to examining the performance differences between HighGear and the new TopGear protocol within our modified version of SCALE-MAMBA. We first looked at two security settings so as to isolate the effect of increasing the Snd_sec parameter alone. Our first setting was the standard SCALE-MAMBA setting of DD_sec = ZK_sec $=$ Snd $\_$sec $=40$, our second was the more secure setting of DD_sec $=$ ZK_sec $=40$ and Snd_sec $=128$.

There are two main parameters in SCALE-MAMBA one can tweak which affect triple production; i) the number of threads devoted to executing the zero-knowledge proofs and ii) the number of threads devoted to taking the output of these proofs and producing triples. We call these two values $t_{\mathrm{ZK}}$ and $t_{\mathrm{Tr}}$; we chose $t_{\mathrm{ZK}}, t_{\mathrm{Tr}} \in\{1,2,4,8\}$ in the experiments. We focus here on triple production for simplicity, a similar situation to that described below occurs in the case of bit production. We examine memory consumption and triple production in these settings so as to see the effect of changing Snd_sec. After this we examine increasing all the security parameters, and the effect this has on memory and triple production.

Memory Consumption: We see from Table 1 that the parameters in TopGear for the underlying FHE scheme are generally identical to those in HighGear, the only difference being when the extra soundness slack in HighGear compared to TopGear is not counter balanced by size of the ciphertext modulus. However, the real effect of TopGear comes in the amount of data one can simultaneously process. Running the implementation in SCALE-MAMBA for HighGear one sees immediately that memory usage is a main constraint of the system.

A rough (under-) estimation of the memory requirements of the ZKPoKs in HighGear and TopGear can be given by the sizes of the input and auxiliary ciphertexts of the ZKPoK. A single ciphertext can be represented by (roughly) $\phi(m) \cdot \log _{2}\left(p_{0} \cdot p_{1}\right)$ bits. There are $U$ input ciphertexts and $V$ auxiliary ciphertexts per player (where $V$ is set to $2 \cdot U-1$ in HighGear). Hence, the total number of bits required to process a ZKPoK is at least $(U+V) \cdot n \cdot \phi(m) \cdot \log _{2}\left(p_{0} \cdot p_{1}\right)$.

Now in HighGear we need to take $U=$ Snd_sec, which is what limits the applicability of large soundness security parameters in the implementations of HighGear. Meanwhile, TopGear can take $V=($ Snd_sec +2$) / \log _{2}(2 \cdot N+1)$, and have arbitrary choice on $U$, although in practice we select $U=2 \cdot V$. For the ZKPoKs for the encryptions of $\alpha$ we have $U=1$ and set $V=16$, simply to reduce memory costs, and then repeat the TopGear proof Snd_sec/16 times.

$\sqrt[8 \text { https://bitbucket.org/malb/lwe-estimator }]{ }$ 
Thus, all other things being equal (which Table 1 gives evidence for) TopGear should reduce the memory footprint by a factor of roughly $\log _{2}(2 \cdot N+1)$. For the range of $N$ under consideration (i.e. 8192 to 32768 ) this gives a memory saving of a factor of between 14 and 16. A similar saving occurs in the amount of data which needs to be transferred when executing the ZKPoK. Note that this is purely the saving for running the zero-knowledge proofs, the overall effect on the memory consumption of the preprocessing will be much less, as that will also include the memory needed to store the output of this offline process.

To see this in practice we examined the memory consumption of running HighGear and TopGear with the above settings (of DD_sec $=$ ZK_sec $=$ Snd $\_s e c=40$, and DD_sec $=$ ZK_sec $=40$, Snd $\_s e c=128$ ) the results being given in Tables 2 and 3 in the Appendix. We give the percentage memory consumption (given in terms of the percentage maximum resident set size obtained from /usr/bin/time $-v$ ). This is the maximum percentage memory consumed by the whole system when producing two million multiplication triples only. This value can vary from run to run as the different threads allocate and de-allocate memory, thus figures will inevitably vary. However, they do give an indication of memory overall consumption in a given configuration.

We find that for HighGear with the higher security parameters we are unable to perform some experiments $\left(t_{\mathrm{ZK}}>\right.$ 2) due to memory consumption producing an abort of the SCALE-MAMBA system. We see immediately that with TopGear the memory consumption drops by a factor $3-7$ for identical security parameters. Furthermore, we are able to cope with a much larger value for the security parameter Snd_sec and all our considered number of threads of the ZKPoK implementation. Even when running $t_{\mathrm{Tr}}=t_{\mathrm{ZK}}=8$ and Snd_sec $=128$ we still only utilize $70 \%$ of memory.

Triple Production Throughput: We now turn to looking at throughput of the overall triple production process where the metric to look at is the average time per triple. However due to the set up costs, (e.g. producing the zeroknowledge proofs for the ciphertext encrypting the MAC key $\alpha$ ) this average time decreases as one runs the system. In the Appendix we provide graphs to show how this average time decreases as more triples are produced for various settings. The spikes in these graphs are due the main triple production threads having to wait for the zero-knowledge threads to complete a zero-knowledge proof before proceeding. Thus a spike indicates a waiting period for a zeroknowledge proofs to complete. As the number of proof threads increases ( $t_{\mathrm{ZK}}$ increases), the effect of these spikes becomes less pronounced. To show the difference between HighGear and TopGear we keep the same $y$-axis in each graph.

We now look at the average number of triples per second we could obtain for the various settings, after computing two million triples ( see Tables 4 and 5 in the Appendix for a summary). The TopGear protocol produces, all other parameters being equal, $2-5$ times as many triples per second than HighGear. This improvement is due to reduced memory consumption ( $U$ and $V$ are smaller), but also because the ratio of $V$ to $U$ in HighGear is larger than that in TopGear ( 2 vs $1 / 2$ in general). In both security settings the TopGear protocol works best when we have $t_{\mathrm{Tr}} \geq 2$.

Recommendations: Given that $2^{- \text {Snd_sec }}$ represents the probability that an adversary can pass of an invalid ZKPoK as valid, the default SCALE-MAMBA v1.2 setting of Snd_sec $=40$ is arguably too low. Thus increasing it to 128 seems definitely prudent.

As mentioned above we also recommend using higher values for ZK_sec and DD_sec. Despite these measuring statistical distances, and hence can be arguably smaller than Snd_sec, in practice they measure the statistical distance of distributions of coefficients from uniformly random. Each ZKPoK/distributed decryption produces tens of thousands of such coefficients, and thus having DD_sec $=$ ZK_sec $=40$ is also probably too low.

We therefore also give some experimental results using TopGear for settings of DD_sec $=$ ZK_sec $=80$, Snd_sec $=$ 128. and DD_sec $=$ ZK_sec $=$ Snd $\_$sec $=128$. Again we focus on the two party case with a plaintext prime of 128 bits in length (with results giving in Tables 6 and 7 in the Appendix). We see that with DD_sec $=$ ZK_sec $=80$ we obtain a triple throughput which is often more than twice that of what SCALE-MAMBA v1.2 achieves using HighGear and DD_sec $=$ ZK_sec $=$ Snd_sec $=40$. On the other hand with DD_sec $=$ ZK_sec $=128$ the performance improvement is less pronounced (improvement by a factor $1.5-2$ ), although still significant. We believe that DD_sec $=$ ZK_sec $=80$ gives a suitable compromise between security and performance.

Also notice in the tables the high memory consumption in the case of $t_{\mathrm{Tr}}=1$ and $t_{\mathrm{ZK}}=8$ compared to (say) $t_{\mathrm{Tr}}=2$ and $t_{\mathrm{ZK}}=8$. This is because in this case memory is increasing as the validated ciphertexts are being produced by the eight zero-knowledge proof threads faster than the single triple production thread can process them. 


\section{Acknowledgments}

We thank Ivan Damgård for his helpful comments. The work of Carsten has been done at Bar Ilan University, Israel. This work has been supported by the BIU Center for Research in Applied Cryptography and Cyber Security in conjunction with the Israel National Cyber Bureau in the Prime Minister's Office, in part by the European Research Council (ERC) under the European Union's Horizon 2020 research and innovation programme under grant agreement No. 669255 (MPCPRO), in part by ERC Advanced Grant ERC-2015-AdG-IMPaCT, by the Defense Advanced Research Projects Agency (DARPA) and Space and Naval Warfare Systems Center, Pacific (SSC Pacific) under contract No. N66001-15-C-4070, and by the FWO under an Odysseus project GOH9718N.

\section{References}

1. Alkim, E., Ducas, L., Pöppelmann, T., Schwabe, P.: Post-quantum key exchange - A New Hope. In: Holz, T., Savage, S. (eds.) 25th USENIX Security Symposium, USENIX Security 16, Austin, TX, USA, August 10-12, 2016. pp. 327-343. USENIX Association (2016), https://www.usenix.org/conference/usenixsecurity16/technical-sessions/ presentation/alkim

2. Aly, A., Keller, M., Orsini, E., Rotaru, D., Scholl, P., Smart, N.P., Wood, T.: SCALE-MAMBA v1.2: Documentation (2018), https://homes.esat.kuleuven.be/ nsmart/SCALE/Documentation.pdf

3. Baum, C., Bootle, J., Cerulli, A., del Pino, R., Groth, J., Lyubashevsky, V.: Sub-linear lattice-based zero-knowledge arguments for arithmetic circuits. In: Shacham, H., Boldyreva, A. (eds.) Advances in Cryptology - CRYPTO 2018, Part II. Lecture Notes in Computer Science, vol. 10992, pp. 669-699. Springer, Heidelberg, Germany, Santa Barbara, CA, USA (Aug 19-23, 2018)

4. Baum, C., Damgård, I., Larsen, K.G., Nielsen, M.: How to prove knowledge of small secrets. In: Robshaw, M., Katz, J. (eds.) Advances in Cryptology - CRYPTO 2016, Part III. Lecture Notes in Computer Science, vol. 9816, pp. 478-498. Springer, Heidelberg, Germany, Santa Barbara, CA, USA (Aug 14-18, 2016)

5. Baum, C., Lyubashevsky, V.: Simple amortized proofs of shortness for linear relations over polynomial rings. Cryptology ePrint Archive, Report 2017/759 (2017), http://eprint.iacr.org/2017/759

6. Ben-Or, M., Goldwasser, S., Kilian, J., Wigderson, A.: Multi-prover interactive proofs: How to remove intractability assumptions. In: 20th Annual ACM Symposium on Theory of Computing. pp. 113-131. ACM Press, Chicago, IL, USA (May 2-4, 1988)

7. Bendlin, R., Damgård, I., Orlandi, C., Zakarias, S.: Semi-homomorphic encryption and multiparty computation. In: Paterson, K.G. (ed.) Advances in Cryptology - EUROCRYPT 2011. Lecture Notes in Computer Science, vol. 6632, pp. 169-188. Springer, Heidelberg, Germany, Tallinn, Estonia (May 15-19, 2011)

8. Benhamouda, F., Camenisch, J., Krenn, S., Lyubashevsky, V., Neven, G.: Better zero-knowledge proofs for lattice encryption and their application to group signatures. In: Sarkar, P., Iwata, T. (eds.) Advances in Cryptology - ASIACRYPT 2014, Part I. Lecture Notes in Computer Science, vol. 8873, pp. 551-572. Springer, Heidelberg, Germany, Kaoshiung, Taiwan, R.O.C. (Dec 7-11, 2014)

9. Benhamouda, F., Krenn, S., Lyubashevsky, V., Pietrzak, K.: Efficient zero-knowledge proofs for commitments from learning with errors over rings. In: Pernul, G., Ryan, P.Y.A., Weippl, E.R. (eds.) ESORICS 2015: 20th European Symposium on Research in Computer Security, Part I. Lecture Notes in Computer Science, vol. 9326, pp. 305-325. Springer, Heidelberg, Germany, Vienna, Austria (Sep 21-25, 2015)

10. Brakerski, Z., Gentry, C., Vaikuntanathan, V.: (Leveled) fully homomorphic encryption without bootstrapping. In: Goldwasser, S. (ed.) ITCS 2012: 3rd Innovations in Theoretical Computer Science. pp. 309-325. Association for Computing Machinery, Cambridge, MA, USA (Jan 8-10, 2012)

11. Brakerski, Z., Vaikuntanathan, V.: Efficient fully homomorphic encryption from (standard) LWE. In: Ostrovsky, R. (ed.) 52nd Annual Symposium on Foundations of Computer Science. pp. 97-106. IEEE Computer Society Press, Palm Springs, CA, USA (Oct 22-25, 2011)

12. Cramer, R., Damgård, I.: On the amortized complexity of zero-knowledge protocols. In: Halevi, S. (ed.) Advances in Cryptology - CRYPTO 2009. Lecture Notes in Computer Science, vol. 5677, pp. 177-191. Springer, Heidelberg, Germany, Santa Barbara, CA, USA (Aug 16-20, 2009)

13. Cramer, R., Damgård, I., Xing, C., Yuan, C.: Amortized complexity of zero-knowledge proofs revisited: Achieving linear soundness slack. In: Coron, J., Nielsen, J.B. (eds.) Advances in Cryptology - EUROCRYPT 2017, Part I. Lecture Notes in Computer Science, vol. 10210, pp. 479-500. Springer, Heidelberg, Germany, Paris, France (Apr 30 - May 4, 2017)

14. Damgård, I., Keller, M., Larraia, E., Pastro, V., Scholl, P., Smart, N.P.: Practical covertly secure MPC for dishonest majority - or: Breaking the SPDZ limits. In: Crampton, J., Jajodia, S., Mayes, K. (eds.) ESORICS 2013: 18th European Symposium on Research in Computer Security. Lecture Notes in Computer Science, vol. 8134, pp. 1-18. Springer, Heidelberg, Germany, Egham, UK (Sep 9-13, 2013) 
15. Damgård, I., Pastro, V., Smart, N.P., Zakarias, S.: Multiparty computation from somewhat homomorphic encryption. In: SafaviNaini, R., Canetti, R. (eds.) Advances in Cryptology - CRYPTO 2012. Lecture Notes in Computer Science, vol. 7417, pp. 643-662. Springer, Heidelberg, Germany, Santa Barbara, CA, USA (Aug 19-23, 2012)

16. del Pino, R., Lyubashevsky, V.: Amortization with fewer equations for proving knowledge of small secrets. In: Katz, J., Shacham, H. (eds.) Advances in Cryptology - CRYPTO 2017, Part III. Lecture Notes in Computer Science, vol. 10403, pp. 365-394. Springer, Heidelberg, Germany, Santa Barbara, CA, USA (Aug 20-24, 2017)

17. del Pino, R., Lyubashevsky, V., Seiler, G.: Short discrete log proofs for FHE and ring-LWE ciphertexts. In: Lin, D., Sako, K. (eds.) PKC 2019: 22nd International Conference on Theory and Practice of Public Key Cryptography, Part I. Lecture Notes in Computer Science, vol. 11442, pp. 344-373. Springer, Heidelberg, Germany, Beijing, China (Apr 14-17, 2019)

18. Ducas, L., Durmus, A., Lepoint, T., Lyubashevsky, V.: Lattice signatures and bimodal Gaussians. In: Canetti, R., Garay, J.A. (eds.) Advances in Cryptology - CRYPTO 2013, Part I. Lecture Notes in Computer Science, vol. 8042, pp. 40-56. Springer, Heidelberg, Germany, Santa Barbara, CA, USA (Aug 18-22, 2013)

19. Ducas, L., Kiltz, E., Lepoint, T., Lyubashevsky, V., Schwabe, P., Seiler, G., Stehlé, D.: CRYSTALS-Dilithium: A lattice-based digital signature scheme. IACR Transactions on Cryptographic Hardware and Embedded Systems 2018(1), 238-268 (2018), https://tches.iacr.org/index.php/TCHES/article/view/839

20. Gentry, C., Halevi, S., Smart, N.P.: Better bootstrapping in fully homomorphic encryption. In: Fischlin, M., Buchmann, J., Manulis, M. (eds.) PKC 2012: 15th International Conference on Theory and Practice of Public Key Cryptography. Lecture Notes in Computer Science, vol. 7293, pp. 1-16. Springer, Heidelberg, Germany, Darmstadt, Germany (May 21-23, 2012)

21. Gentry, C., Halevi, S., Smart, N.P.: Fully homomorphic encryption with polylog overhead. In: Pointcheval, D., Johansson, T. (eds.) Advances in Cryptology - EUROCRYPT 2012. Lecture Notes in Computer Science, vol. 7237, pp. 465-482. Springer, Heidelberg, Germany, Cambridge, UK (Apr 15-19, 2012)

22. Gentry, C., Halevi, S., Smart, N.P.: Homomorphic evaluation of the AES circuit. In: Safavi-Naini, R., Canetti, R. (eds.) Advances in Cryptology - CRYPTO 2012. Lecture Notes in Computer Science, vol. 7417, pp. 850-867. Springer, Heidelberg, Germany, Santa Barbara, CA, USA (Aug 19-23, 2012)

23. Halevi, S., Shoup, V.: Algorithms in HElib. In: Garay, J.A., Gennaro, R. (eds.) Advances in Cryptology - CRYPTO 2014, Part I. Lecture Notes in Computer Science, vol. 8616, pp. 554-571. Springer, Heidelberg, Germany, Santa Barbara, CA, USA (Aug 17-21, 2014)

24. Keller, M., Orsini, E., Scholl, P.: MASCOT: Faster malicious arithmetic secure computation with oblivious transfer. In: Weippl, E.R., Katzenbeisser, S., Kruegel, C., Myers, A.C., Halevi, S. (eds.) ACM CCS 2016: 23rd Conference on Computer and Communications Security. pp. 830-842. ACM Press, Vienna, Austria (Oct 24-28, 2016)

25. Keller, M., Pastro, V., Rotaru, D.: Overdrive: Making SPDZ great again. In: Nielsen, J.B., Rijmen, V. (eds.) Advances in Cryptology - EUROCRYPT 2018, Part III. Lecture Notes in Computer Science, vol. 10822, pp. 158-189. Springer, Heidelberg, Germany, Tel Aviv, Israel (Apr 29 - May 3, 2018)

26. Lyubashevsky, V.: Fiat-Shamir with aborts: Applications to lattice and factoring-based signatures. In: Matsui, M. (ed.) Advances in Cryptology - ASIACRYPT 2009. Lecture Notes in Computer Science, vol. 5912, pp. 598-616. Springer, Heidelberg, Germany, Tokyo, Japan (Dec 6-10, 2009)

\section{A Parameter Size Table}

See Table 1 for the various FHE parameter sizes for our different security levels. 


\begin{tabular}{|c|c|c|c|c|c|c|c|c|c|c|c|c|c|}
\hline & & & & & & \multicolumn{3}{|c|}{ HighGear } & \multicolumn{5}{|c|}{ TopGear } \\
\hline & $\log _{2} p$ & $\kappa$ & DD_sec & Snd_sec & ZK_sec & $N$ & $\log _{2} p_{0}$ & $\log _{2} p_{1}$ & $N$ & $U$ & $V$ & $\log _{2} p_{0}$ & $\log _{2} p_{1}$ \\
\hline & 64 & 80 & 40 & 40 & 40 & 8192 & 177 & 114 & 8192 & 6 & 3 & 176 & 115 \\
\hline & 64 & 80 & 40 & 80 & 40 & 8192 & 177 & 114 & 8192 & 12 & 6 & 176 & 115 \\
\hline & 64 & 80 & 40 & 128 & 40 & 8192 & 177 & 114 & 8192 & 18 & 9 & 176 & 115 \\
\hline & 64 & 80 & 40 & 80 & 80 & 8192 & 177 & 144 & 8192 & 12 & 6 & 176 & 115 \\
\hline & 64 & 80 & 40 & 128 & 80 & 16384 & 177 & 174 & 8192 & 18 & 9 & 167 & 115 \\
\hline & 64 & 80 & 80 & 40 & 40 & 16384 & 218 & 163 & 16384 & 6 & 3 & 217 & 164 \\
\hline & 64 & 80 & 80 & 80 & 40 & 16384 & 218 & 163 & 16384 & 12 & 6 & 217 & 164 \\
\hline & 64 & 80 & 80 & 128 & 40 & 16384 & 218 & 163 & 16384 & 18 & 9 & 217 & 164 \\
\hline & 64 & 80 & 80 & 80 & 80 & 16384 & 218 & 163 & 16384 & 12 & 6 & 217 & 164 \\
\hline & 64 & 80 & 80 & 128 & 80 & 16384 & 218 & 173 & 16384 & 18 & 9 & 217 & 164 \\
\hline & 64 & 80 & 128 & 40 & 40 & 16384 & 266 & 205 & 16384 & 6 & 3 & 265 & 206 \\
\hline & 64 & 80 & 128 & 80 & $\star$ & 16384 & 266 & 205 & 16384 & 12 & 6 & 265 & 206 \\
\hline & 64 & 80 & 128 & 128 & $\star$ & 16384 & 266 & 205 & 16384 & 18 & 9 & 265 & 206 \\
\hline$\checkmark$ & 64 & 128 & 40 & 40 & 40 & 16384 & 178 & 123 & 16384 & 6 & 3 & 177 & 124 \\
\hline & 64 & 128 & 40 & 80 & 40 & 16384 & 178 & 123 & 16384 & 12 & 6 & 177 & 124 \\
\hline & 64 & 128 & 40 & 128 & 40 & 16384 & 178 & 133 & 16384 & 18 & 9 & 177 & 124 \\
\hline & 64 & 128 & 40 & 80 & 80 & 16384 & 178 & 153 & 16384 & 12 & 6 & 177 & 124 \\
\hline & 64 & 128 & 40 & 128 & 80 & 16384 & 178 & 173 & 16384 & 18 & 9 & 177 & 124 \\
\hline & 64 & 128 & 80 & 40 & 40 & 16384 & 218 & 163 & 16384 & 6 & 3 & 217 & 164 \\
\hline & 64 & 128 & 80 & 80 & 40 & 16384 & 218 & 163 & 16384 & 12 & 6 & 217 & 164 \\
\hline & 64 & 128 & 80 & 128 & 40 & 16384 & 218 & 163 & 16384 & 18 & 9 & 217 & 164 \\
\hline & 64 & 128 & 80 & 80 & 80 & 16384 & 218 & 163 & 16384 & 12 & 6 & 217 & 164 \\
\hline$\checkmark \checkmark \checkmark$ & 64 & 128 & 80 & 128 & 80 & 16384 & 218 & 173 & 16384 & 18 & 9 & 217 & 164 \\
\hline & 64 & 128 & 128 & 40 & $\star$ & 32768 & 266 & 205 & 32768 & 6 & 3 & 266 & 205 \\
\hline & 64 & 128 & 128 & 80 & * & 32768 & 266 & 205 & 32768 & 10 & 5 & 266 & 205 \\
\hline & 64 & 128 & 128 & 128 & $\star$ & 32768 & 266 & 205 & 32768 & 16 & 8 & 266 & 205 \\
\hline$\checkmark \checkmark \checkmark$ & 64 & 128 & 128 & 128 & 128 & 32768 & 266 & 225 & 32768 & 16 & 8 & 266 & 205 \\
\hline & 128 & 80 & 40 & 40 & 40 & 16384 & 305 & 186 & 16384 & 6 & 3 & 305 & 186 \\
\hline & 128 & 80 & 40 & 80 & $\star$ & 16384 & 305 & 186 & 16384 & 12 & 6 & 305 & 186 \\
\hline & 128 & 80 & 40 & 128 & $\star$ & 16384 & 305 & 186 & 16384 & 18 & 9 & 305 & 186 \\
\hline & 128 & 80 & 80 & 40 & $\star$ & 16384 & 345 & 226 & 16384 & 6 & 3 & 345 & 226 \\
\hline & 128 & 80 & 80 & 80 & $\star$ & 16384 & 345 & 226 & 16384 & 12 & 6 & 345 & 226 \\
\hline & 128 & 80 & 80 & 128 & $\star$ & 16384 & 345 & 226 & 16384 & 18 & 9 & 345 & 226 \\
\hline & 128 & 80 & 128 & 40 & $\star$ & 16384 & 393 & 268 & 16384 & 6 & 3 & 393 & 268 \\
\hline & 128 & 80 & 128 & 80 & $\star$ & 16384 & 393 & 268 & 16384 & 12 & 6 & 393 & 268 \\
\hline & 128 & 80 & 128 & 128 & $\star$ & 16384 & 393 & 268 & 16384 & 18 & 9 & 393 & 268 \\
\hline$\checkmark$ & 128 & 128 & 40 & 40 & 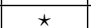 & 32768 & 306 & 185 & 32768 & 6 & 3 & 306 & 185 \\
\hline & 128 & 128 & 40 & 80 & $\star$ & 32768 & 306 & 185 & 32768 & 10 & 5 & 306 & 185 \\
\hline$\checkmark \checkmark$ & 128 & 128 & 40 & 128 & $\star$ & 32768 & 306 & 185 & 32768 & 16 & 8 & 306 & 185 \\
\hline & 128 & 128 & 80 & 40 & $\star$ & 32768 & 346 & 225 & 32768 & 6 & 3 & 346 & 225 \\
\hline & 128 & 128 & 80 & 80 & $\star$ & 32768 & 346 & 225 & 32768 & 10 & 5 & 346 & 225 \\
\hline$\checkmark \checkmark \checkmark$ & 128 & 128 & 80 & 128 & $\star$ & 32768 & 346 & 225 & 32768 & 16 & 8 & 346 & 225 \\
\hline & 128 & 128 & 128 & 40 & $\star$ & 32768 & 394 & 277 & 32768 & 6 & 3 & 394 & 277 \\
\hline & 128 & 128 & 128 & 80 & $\star$ & 32768 & 394 & 277 & 32768 & 10 & 5 & 394 & 277 \\
\hline & 128 & 128 & 128 & 128 & $\star$ & 32768 & 394 & 277 & 32768 & 16 & 8 & 394 & 277 \\
\hline$\checkmark \checkmark$ & 128 & 128 & 128 & 128 & 128 & 32768 & 394 & 277 & 32768 & 16 & 8 & 394 & 277 \\
\hline
\end{tabular}

Table 1. SHE parameters sizes for various security parameters in HighGear and TopGear (two parties). With DD_sec, ZK_sec $\leq$ Snd_sec and DD_sec, ZK_sec, Snd_sec $\in\{40,80,128\}$. The single checkmark for a row shows the default parameters used in SCALE-MAMBA v1.2. Two checkmarks denote the parameters we use in the experiments related to memory and throughput. The rows with three checkmarks show the parameters we would recommend. 


\section{B Experimental Data}

\begin{tabular}{|c||c|c|c|c|}
\hline \multicolumn{1}{|c||}{} & \multicolumn{4}{c|}{$t_{\text {ZK }}$} \\
$t_{\text {Tr }}$ & 1 & 2 & 4 & 8 \\
\hline 1 & 25 & 41 & 68 & 98 \\
2 & 25 & 38 & 68 & 98 \\
4 & 28 & 49 & 75 & 98 \\
8 & 32 & 52 & 81 & 98 \\
\hline DD_sec $=$ ZK_sec $=$ Snd_sec $=40$
\end{tabular}

\begin{tabular}{|c||c|c|c|c|}
\hline \multicolumn{1}{|c||}{} & \multicolumn{5}{c|}{$t_{\text {ZK }}$} \\
$t_{\text {Tr }}$ & 1 & 2 & 4 & 8 \\
\hline 1 & 70 & 98 & - & - \\
2 & 72 & 98 & - & - \\
4 & 73 & 98 & - & - \\
8 & 76 & 98 & - & - \\
DD_sec $=$ ZK_sec $=40$, Snd_sec $=128$
\end{tabular}

Table 2. Percentage memory consumption for HighGear for two players and $\log _{2} p=128$.

\begin{tabular}{c||c|c|c|c|}
\hline \multicolumn{1}{|c||}{} & \multicolumn{4}{c|}{$t_{\mathrm{ZK}}$} \\
$t_{\mathrm{Tr}}$ & 1 & 2 & 4 & 8 \\
\hline 1 & 7 & 9 & 15 & 27 \\
2 & 8 & 10 & 15 & 26 \\
4 & 10 & 12 & 17 & 28 \\
8 & 14 & 17 & 21 & 33 \\
DD_sec $=$ ZK_sec $=$ Snd_sec $=40$
\end{tabular}

\begin{tabular}{|c||c|c|c|c|}
\hline \multicolumn{1}{|c||}{} & \multicolumn{4}{c|}{$t_{\text {ZK }}$} \\
$t_{\text {Tr }}$ & 1 & 2 & 4 & 8 \\
\hline 1 & 11 & 19 & 33 & 63 \\
2 & 12 & 18 & 33 & 64 \\
4 & 14 & 21 & 34 & 64 \\
8 & 16 & 24 & 39 & 70 \\
DD_sec $=$ ZK_sec $=40$, Snd_sec $=128$
\end{tabular}

Table 3. Percentage memory consumption for TopGear for two players and $\log _{2} p=128$.

\begin{tabular}{|c||c|c|c|c|}
\hline \multicolumn{1}{|c||}{} & \multicolumn{4}{c|}{$t_{\text {ZK }}$} \\
$t_{\mathrm{Tr}}$ & 1 & 2 & 4 & 8 \\
\hline 1 & 1503 & 1602 & 1562 & 1335 \\
2 & 1488 & 2347 & 2212 & 1976 \\
4 & 1272 & 1876 & 2150 & 1865 \\
8 & 976 & 1307 & 1464 & 1533 \\
\hline \multicolumn{4}{l|}{ DD_sec $=$ ZK_sec $=$ Snd_sec $=40$}
\end{tabular}

\begin{tabular}{c||c||c|c|c|c|}
\hline \multicolumn{1}{|c||}{} & \multicolumn{5}{c|}{$t_{\text {ZK }}$} \\
$t_{\mathrm{Tr}}$ & 1 & 2 & 4 & 8 \\
\hline 1 & 1240 & 1369 & - & - \\
2 & 1426 & 1834 & - & - \\
4 & 1231 & 1612 & - & - \\
8 & 940 & 1129 & - & - \\
DD_sec $=$ ZK_sec $=40$, Snd_sec $=128$
\end{tabular}

Table 4. Maximum Triples per Second for HighGear for two players and $\log _{2} p=128$, after computing two million triples.

\begin{tabular}{|c|c|c|c|c|}
\hline \multirow[b]{2}{*}{$t_{\mathrm{Tr}}$} & \multicolumn{4}{|c|}{$t_{\text {ZK }}$} \\
\hline & 1 & 2 & 4 & 8 \\
\hline 1 & 2806 & 2829 & 2846 & 2752 \\
\hline 2 & 3809 & 4851 & 4709 & 4540 \\
\hline 4 & 5672 & 6086 & 6692 & 6293 \\
\hline 8 & 4666 & 5635 & 6084 & 5636 \\
\hline
\end{tabular}

\begin{tabular}{c||c|c|c|c|}
\hline \multicolumn{1}{|c||}{} & \multicolumn{4}{c|}{$t_{\text {ZK }}$} \\
$t_{\mathrm{Tr}}$ & 1 & 2 & 4 & 8 \\
\hline 1 & 1743 & 2775 & 2692 & 2569 \\
2 & 4251 & 4622 & 4572 & 4021 \\
4 & 3943 & 3712 & 4955 & 5000 \\
8 & 3265 & 3272 & 5254 & 5041 \\
\hline DD_sec $=$ ZK_sec $=40$, Snd_sec $=128$
\end{tabular}

Table 5. Maximum Triples per Second for TopGear for two players and $\log _{2} p=128$, after computing two million triples. 


\begin{tabular}{|c||c|c|c|c|}
\hline \multicolumn{1}{|c||}{} & \multicolumn{4}{c|}{$t_{\text {ZK }}$} \\
$t_{\mathrm{Tr}}$ & 1 & 2 & 4 & 8 \\
\hline 1 & 12 & 19 & 35 & 75 \\
2 & 13 & 19 & 33 & 65 \\
4 & 15 & 21 & 36 & 67 \\
8 & 18 & 26 & 40 & 76 \\
\hline
\end{tabular}

\begin{tabular}{|c|c|c|c|c|}
\hline \multirow[b]{2}{*}{$t_{\operatorname{Tr}}$} & \multicolumn{4}{|c|}{$t_{\text {ZK }}$} \\
\hline & 1 & 2 & 4 & 8 \\
\hline 1 & $2312(153)$ & $2378(148)$ & $2369(151)$ & $2178(163)$ \\
\hline 2 & $3632(244)$ & 3980 (169) & $3905(176)$ & 3354 (169) \\
\hline 4 & $3260(256)$ & 4709 (251) & 4667 (217) & $3976(213)$ \\
\hline 8 & $2736(280)$ & $3959(302)$ & $4628(316)$ & $3703(241)$ \\
\hline
\end{tabular}

Table 6. Percentage memory consumption and triples per second for TopGear for two players with DD_sec $=$ ZK_sec $=80$ and $\log _{2} p=$ Snd_sec $=128$. We also give (in brackets) the percentage throughput compared to the (low security) standard SCALE-MAMBA v1.2 settings using HighGear.

\begin{tabular}{|c||c|c|c|c|}
\hline \multicolumn{1}{|c||}{} & \multicolumn{4}{c|}{$t_{\text {ZK }}$} \\
$t_{\mathrm{Tr}}$ & 1 & 2 & 4 & 8 \\
\hline 1 & 14 & 21 & 40 & 90 \\
2 & 14 & 22 & 38 & 78 \\
4 & 17 & 24 & 40 & 78 \\
8 & 18 & 28 & 47 & 87 \\
\hline \multicolumn{4}{|c|}{ Memory Consumption }
\end{tabular}

\begin{tabular}{|c||c|c|c|c|}
\hline \multicolumn{1}{|c||}{$t_{\operatorname{Tr}}$} & 1 & 2 & 4 & 8 \\
\hline 1 & $1604(107)$ & $1923(120)$ & $1921(122)$ & $1775(132)$ \\
2 & $2945(198)$ & $3281(139)$ & $3265(147)$ & $2868(145)$ \\
4 & $2605(205)$ & $2923(155)$ & $4046(188)$ & $3427(183)$ \\
8 & $2080(213)$ & $2571(196)$ & $3516(240)$ & $3322(216)$ \\
\hline \multicolumn{5}{|c|}{ Triples per Second } \\
\hline \multicolumn{5}{|c|}{}
\end{tabular}

Table 7. Percentage memory consumption and triples per second for TopGear for two players with $\log _{2} p=$ DD_sec $=$ ZK_sec $=$ Snd_sec $=128$. Again, we also give (in brackets) the percentage throughput compared to the (low security) standard SCALEMAMBA v1.2 settings using HighGear.

\section{Run Time Graphs}

In Figure 4 we provide graphs of the throughput for HighGear in our low security, Snd_sec $=40$, setting, with the comparable graph for TopGear in Figure 5 for two players; given graphs up to the production of 2 million triples. The fact that the graphs are not straight, they have bumps in them, is because the triple production threads are producing triples faster than the ciphertexts can be supplied by the threads doing the ZKPoKs. Thus the triple production threads often need to wait until a ZKPoK has been completed before they can proceed. In Figure 6 and Figure 7 we provide similar graphs of the throughput for HighGear and TopGear in our high security setting Snd_sec $=128$. 

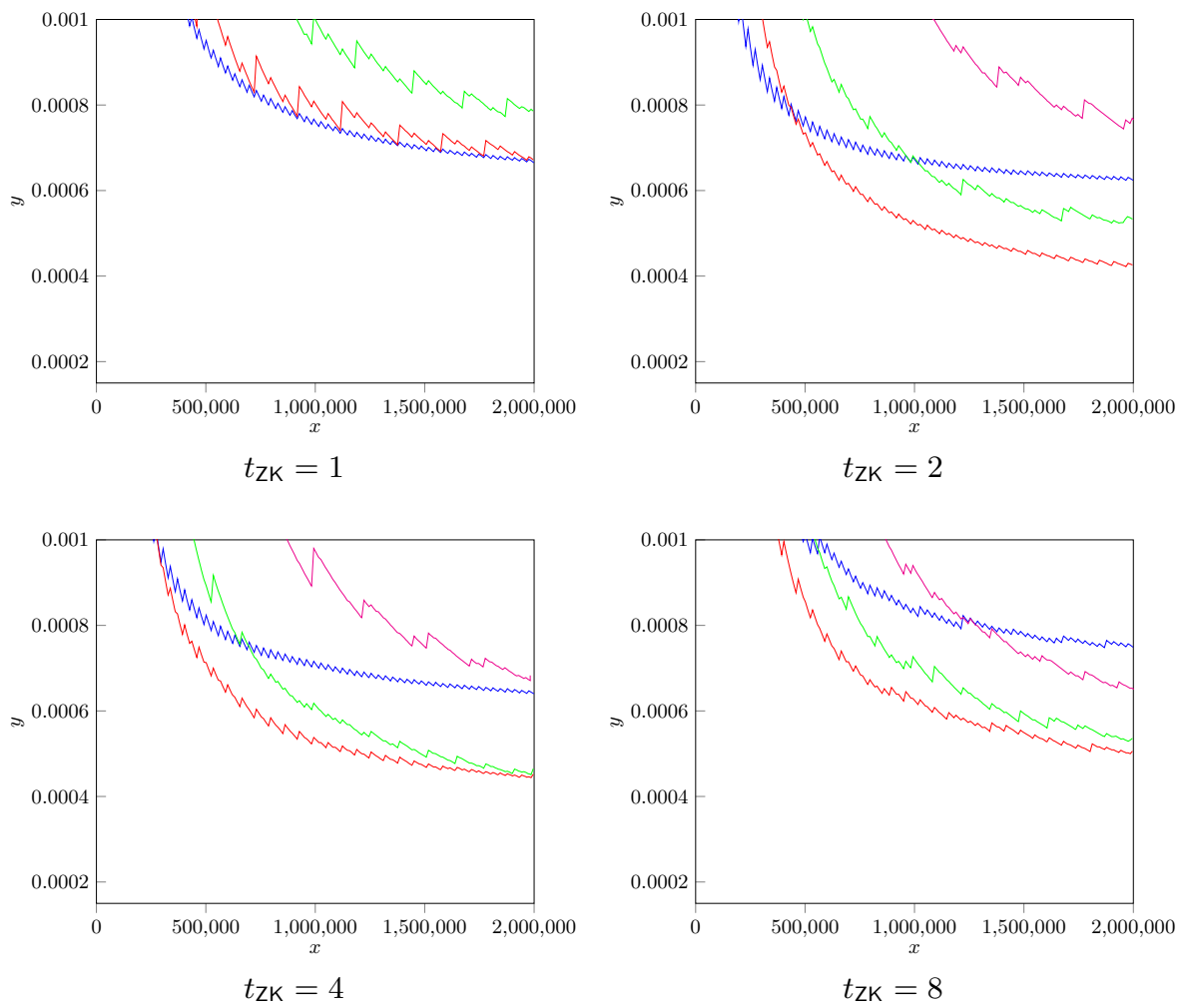

Fig. 4. Average time $y$ to produce a triple given the number of triples that have been produced $x$ for HighGear with parameters DD_sec $=$ ZK_sec $=$ Snd_sec $=40$.

Blue $t_{\mathrm{Tr}}=1$, Red $t_{\mathrm{Tr}}=2$, Green $t_{\mathrm{Tr}}=4$, Magenta $t_{\mathrm{Tr}}=8$ 

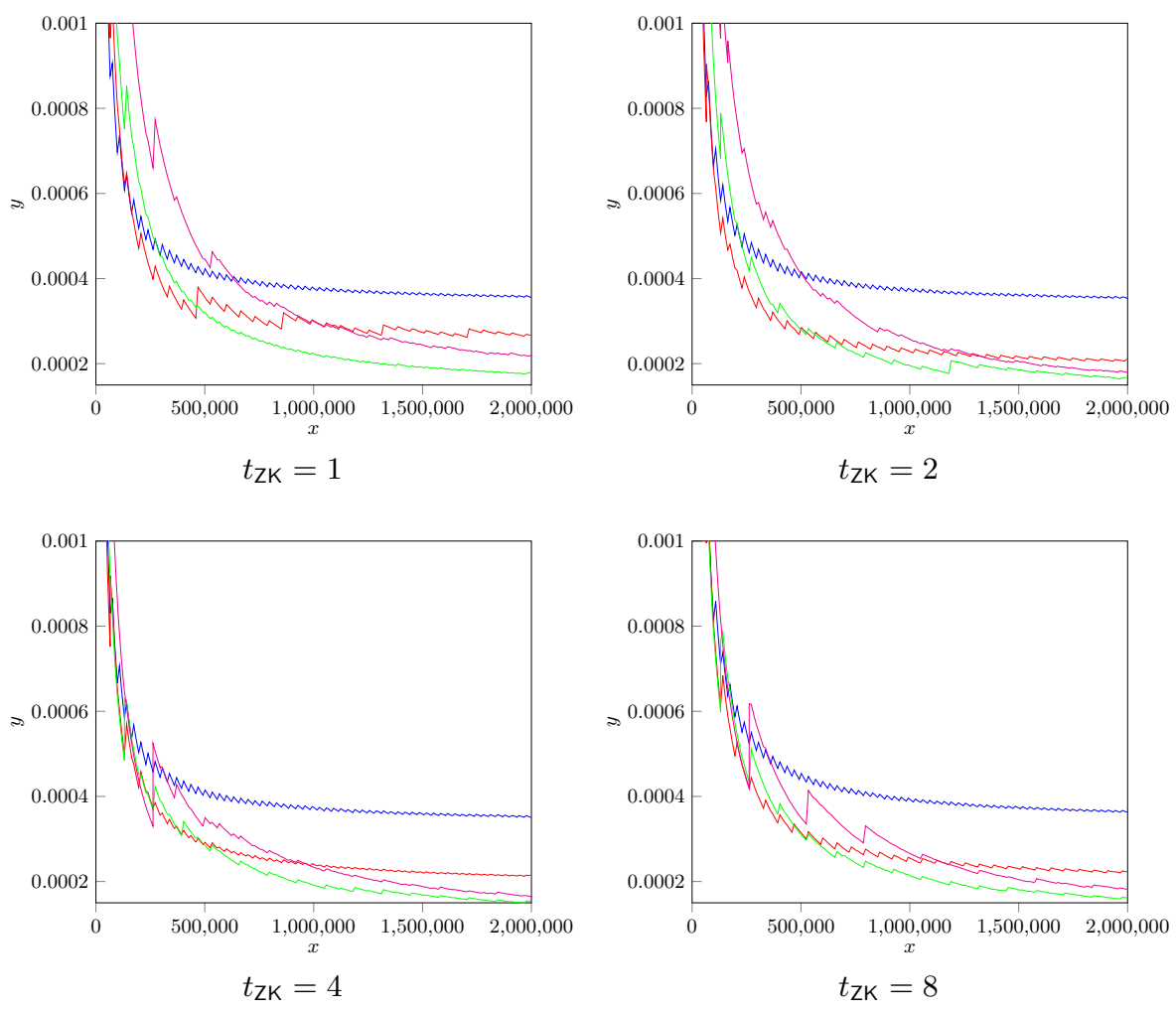

Fig. 5. Average time $y$ to produce a triple given the number of triples that have been produced $x$ for TopGear with parameters DD_sec $=$ ZK_sec $=$ Snd_sec $=40$.

Blue $t_{T r}=1$, Red $t_{T r}=2$, Green $t_{T r}=4$, Magenta $t_{T r}=8$
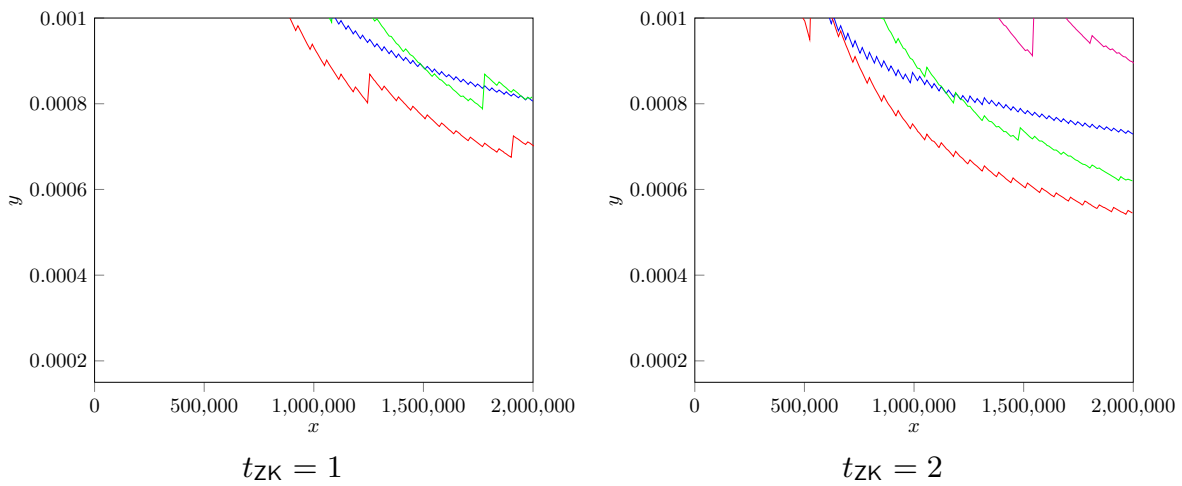

Fig. 6. Average time $y$ to produce a triple given the number of triples that have been produced $x$ for HighGear with parameters DD_sec $=$ ZK_sec $=40$ and Snd_sec $=128$.

Blue $t_{\mathrm{Tr}}=1$, Red $t_{\mathrm{Tr}}=2$, Green $t_{\mathrm{Tr}}=4$, Magenta $t_{\mathrm{Tr}}=8$ 

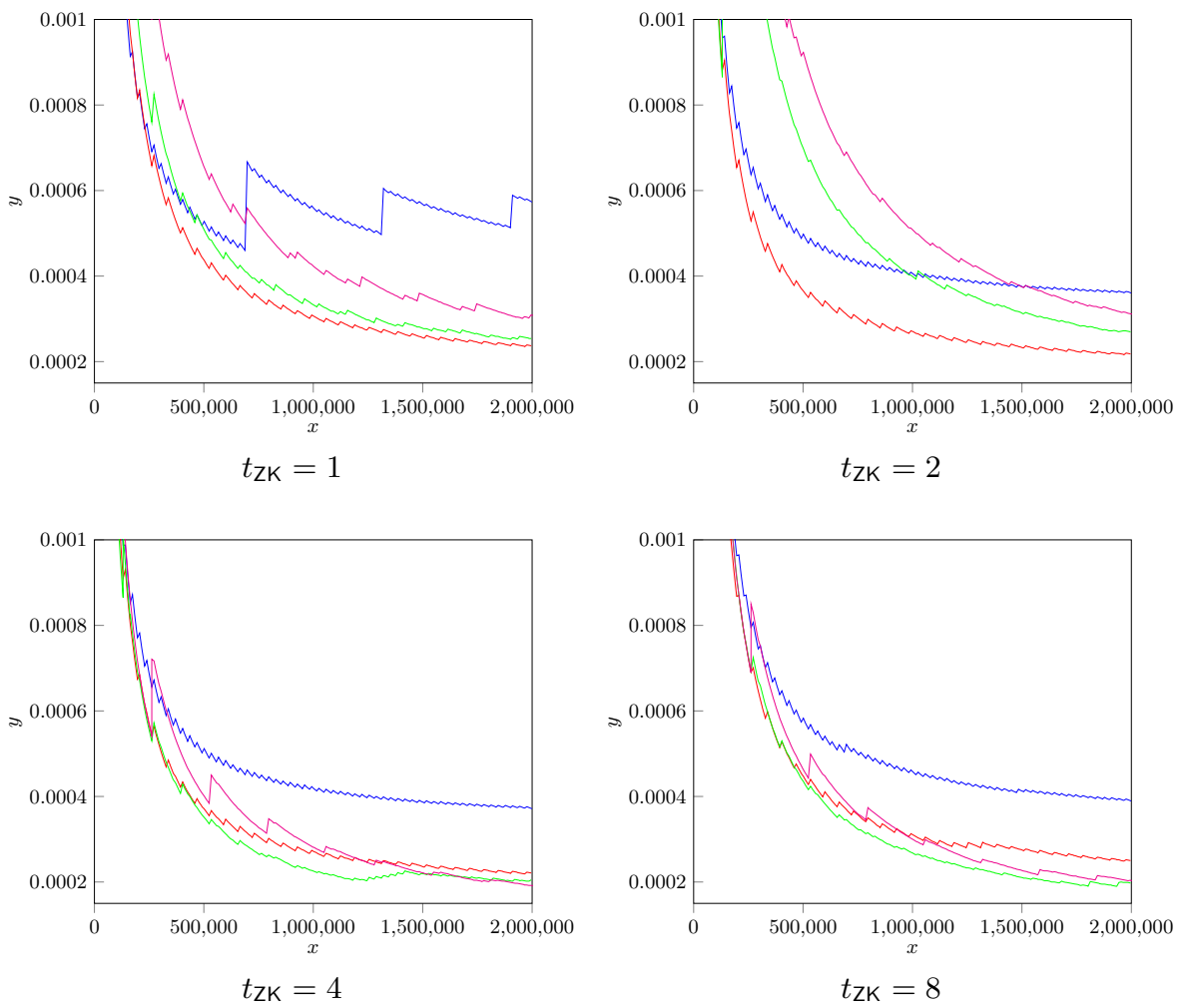

Fig. 7. Average time $y$ to produce a triple given the number of triples that have been produced $x$ for TopGear with parameters DD_sec $=$ ZK_sec $=40$ and Snd_sec $=128$.

Blue $t_{\mathrm{Tr}}=1$, Red $t_{\mathrm{Tr}}=2$, Green $t_{\mathrm{Tr}}=4$, Magenta $t_{\mathrm{Tr}}=8$ 